\title{
The origin of $\mathrm{HI}$-deficiency in galaxies on the outskirts of the Virgo cluster ${ }^{\star}$
}

\section{Companions and uncertainties in distances and deficiencies}

\author{
T. Sanchis ${ }^{1}$, G. A. Mamon ${ }^{2,3}$, E. Salvador-Solé ${ }^{1,4}$, and J. M. Solanes ${ }^{1,4}$ \\ 1 Departament d'Astronomia i Meteorologia, Universitat de Barcelona, Martí i Franquès 1, 08028 Barcelona, Spain \\ e-mail: [tsanchis; eduard; jsolanes]@am.ub.es \\ 2 Institut d'Astrophysique de Paris (CNRS UMR 7095), 98 bis Bd Arago, 75014 Paris, France \\ e-mail: gam@iap.fr \\ 3 GEPI (CNRS UMR 8111), Observatoire de Paris, 92195 Meudon Cedex, France \\ ${ }^{4}$ CER on Astrophysics, Particle Physics, and Cosmology, Universitat de Barcelona, Martí i Franquès 1, \\ 08028 Barcelona, Spain
}

Received 5 August 2003 / Accepted 21 January 2004

\begin{abstract}
The origin of the deficiency in neutral hydrogen of 13 spiral galaxies lying in the outskirts of the Virgo cluster is reassessed. If these galaxies have passed through the core of the cluster, their interstellar gas should have been lost through ram pressure stripping by the hot X-ray emitting gas of the cluster. We analyze the positions of these $\mathrm{H}$ I-deficient and other spiral galaxies in velocity-distance plots, in which we include our compilation of velocity-distance data on 61 elliptical galaxies, and compare with simulated velocity-distance diagrams obtained from cosmological $N$-body simulations. We find that $\sim 20 \%$ relative Tully-Fisher distance errors are consistent with the great majority of the spirals, except for a small number of objects whose positions in the velocity-distance diagram suggest grossly incorrect distances, implying that the Tully-Fisher error distribution function has non-Gaussian wings. Moreover, we find that the distance errors may lead to an incorrect fitting of the TolmanBondi solution that can generate significant errors in the distance and especially the mass estimates of the cluster. We suggest 4 possibilities for the outlying $\mathrm{H}$ I-deficient spirals (in decreasing frequency): 1) they have large relative distance errors and are in fact close enough (at distances between 12.7 and $20.9 \mathrm{Mpc}$ from us) to the cluster to have passed through its core and seen their gas removed by ram pressure stripping; 2) their gas is converted to stars by tidal interactions with other galaxies; 3) their gas is heated during recent mergers with smaller galaxies; and 4) they are not truly H I-deficient (e.g. S0/a misclassified as Sa).
\end{abstract}

Key words. galaxies: clusters: individual: Virgo - galaxies: evolution - galaxies: ISM - cosmology: distance scale methods: $N$-body simulations

\section{Introduction}

Over the last thirty years, substantial work has been carried out to quantify the effects of the environment on the properties of galaxies, i.e. to distinguish the characteristics of galaxies in dense, aggressive regions such as clusters, especially cluster cores, and low density zones away from clusters and groups. The nearby Virgo cluster is an excellent candidate for such studies, because its proximity allows 1) an easy comparison with field galaxies and 2) the measurement of distances independent of radial velocity, providing three-dimensional information on cluster properties (e.g. Young \& Currie 1995;

\footnotetext{
Send offprint requests to: T. Sanchis,

e-mail: tsanchis@am.ub.es

* Appendix A is only available in electronic form at http://www. edpsciences.org
}

Gavazzi et al. 1999; Solanes et al. 2002) impossible to obtain in other clusters.

One particular characteristic of spiral galaxies is their neutral hydrogen (HI) gas content. It has been known for some time that a class of H I-deficient galaxies exists in the centers of clusters, such as the Virgo cluster (Chamaraux et al. 1980), and their deficiency, normalized to their optical diameter and morphological type, is anti-correlated with their clustercentric distance (Haynes \& Giovanelli 1986; Solanes et al. 2001).

Chamaraux et al. (1980) considered ram pressure stripping of interstellar atomic hydrogen by the hot diffuse intracluster gas, first proposed by Gunn \& Gott (1972), as one cause of the H I deficiency pattern of Virgo spirals. For galaxies moving face-on, ram pressure scales as the intracluster gas density times the square of the galaxy's velocity relative to this intracluster gas, so the role of ram pressure stripping was 
strengthened by the correlation of H I deficiency with the X-ray luminosity of clusters found by Giovanelli \& Haynes (1985; see however Solanes et al. 2001) and by the presence of truncated H I disks observed by Cayatte et al. (1990).

It hence came as a surprise when Solanes et al. (2002) discovered spiral galaxies, whose hydrogen content was deficient relative to other spiral galaxies of the same morphological type, far away from the core of Virgo, with several deficient spirals in the foreground of Virgo and particularly one group of spirals 7 to $10 \mathrm{Mpc}$ (depending on the adopted distance to Virgo) behind the cluster with one-third of its 15 spirals displaying $\mathrm{HI}$ deficiencies deviating by more than $3 \sigma$ from normalcy.

In a followup study, Sanchis et al. (2002) explored the possibility that the group of deficient spirals behind Virgo might have plunged into the cluster core a few Gyr ago and lost their gas by interacting with the hot intracluster medium. Sanchis et al. applied a simple dynamical model that considered not only the classical deduction of the virgocentric infalling regime, but also the trajectories of galaxies that had already crossed once the center of the core. This model succeeded in explaining the overall characteristics of the observed velocity-distance diagram, including the envelope of the virialized cluster drawn by galaxies on first rebound. With the available data, they argued, from a statistical point of view, that one could not discard the possibility that some of the gas-deficient objects, including the background group in the outskirts of the Virgo Cluster Region, were not newcomers, but have passed through the cluster and lost their gas by ram pressure stripping.

We (Mamon et al. 2004) have recently estimated the maximum radius out to where galaxies falling into a cluster can bounce out. We found that this maximum rebound radius corresponds to 1 to 2.5 cluster virial radii. After estimating the virial radius of the Virgo cluster using X-ray observations, we concluded that the HI-deficient galaxies located in the foreground or background of the Virgo cluster appear to lie much farther from the center of the cluster than these maximum distances, and therefore that ram pressure stripping cannot account for the H I-deficiency of these outlying galaxies if their distances are correct.

In this paper, we investigate in further detail the origin of the H I-deficiency in the spiral galaxies that appear too far from the Virgo cluster to have suffered ram pressure stripping of their interstellar gas, by 1) adding to our four-dimensional (angular position, distance and radial velocity) sample of spiral galaxies, our compilation of the literature for elliptical galaxies towards Virgo, leading us to identify more precisely the density of the environment of the background deficient galaxies and estimate more accurately the distance to the center of Virgo, which is a crucial ingredient for the dynamical infall model, and 2) using cosmological $N$-body simulations that have the advantages of their lack of observational errors, completeness, and of defining a realistic infall pattern.

The sample of elliptical galaxies is described in Sect. 2. In Sect. 3, after describing the cosmological $N$-body simulations used here, we compare the simulated and observational samples to evaluate the influence of errors in distance estimates. Finally in Sect. 4, we discuss possible explanations for the $\mathrm{HI}$ deficiency of the outlying galaxies.
Table 1. Datasets contributing to elliptical sample.

\begin{tabular}{lcccc}
\hline \hline Method $^{a}$ & Authors $^{b}$ & Band & $\begin{array}{c}\text { Rel. accuracy } \\
\text { (rms) }\end{array}$ & Number \\
\hline $\mathrm{FP}$ & $\mathrm{G}$ & $H$ & 0.41 & 41 \\
$L-n$ & $\mathrm{Y}_{L}$ & $B_{J}$ & 0.54 & 9 \\
$R-n$ & $\mathrm{Y}_{R}$ & $B_{J}$ & 0.54 & 14 \\
$\mathrm{SBF}$ & $\mathrm{T}$ & $I$ & 0.18 & 31 \\
$\mathrm{SBF}$ & $\mathrm{J}_{I}$ & $I$ & 0.07 & 6 \\
$\mathrm{SBF}$ & $\mathrm{J}_{K}$ & $K^{\prime}$ & 0.17 & 7 \\
$\mathrm{SBF}$ & $\mathrm{N}$ & $I$ & 0.15 & 15 \\
$\mathrm{GCLF}$ & $\mathrm{K}$ & $V-I$ & 0.07 & 8 \\
\hline
\end{tabular}

${ }^{a} \mathrm{FP}=$ Fundamental Plane; $L-n, R-n=$ Luminosity $/$ Sérsic shape and Radius / Sérsic shape relations; SBF = Surface Brightness Fluctuations; GCLF $=$ Globular Cluster Luminosity Function. ${ }^{b} \mathrm{G}=$ Gavazzi et al. (1999); $\mathrm{T}=$ Tonry et al. (2001); $\mathbf{J}_{I}, \mathbf{J}_{K}=$ Jensen et al. (1998); N = Neilsen \& Tsvetanov (2000); K = Kundu \& Whitmore (2001); $\mathrm{Y}_{L}, \mathrm{Y}_{R}=$ Young \& Currie (1995).

\section{The inclusion of ellipticals into the Virgo galaxy sample}

Using only late-type spirals, Sanchis et al. (2002) applied a spherical infall model to the velocity field of the Virgo Cluster Region and obtained two possible distances of 20 and $21 \mathrm{Mpc}$. Other distance estimates to the Virgo cluster, based upon spiral galaxies, produced distances in the range from $16 \mathrm{Mpc}$ (Tully $\&$ Shaya 1984) to $21 \mathrm{Mpc}$ (Ekholm et al. 2000).

An alternative way to estimate the cluster distance is by using the early-type components of the cluster, as they are thought to be better tracers of the center of the cluster due to morphological segregation (see e.g. Ferguson \& Binggeli 1994). To do so, we have collected as many as possible earlytype galaxies (E's, dE's and S0's) with redshift-independent distance measurements. Heliocentric velocities were extracted from the LEDA catalog of the HyperLEDA database and converted to the Local Group reference frame, while the direct distant measurements are obtained from a variety of sources.

Table 1 summarizes the distance datasets on the early-type galaxies towards Virgo. Although different authors use different methods with different accuracy, we have not found systematic deviations between them, and furthermore found that the spread in differences of the distance moduli was consistent with the quoted errors, for all sets of early-type galaxies common to two authors. Therefore, we estimated a global distance modulus $\bar{d}$ for each galaxy, using the maximum likelihood estimator (e.g. Bevington \& Robinson 1992)

$\bar{d}=\frac{\sum_{i} d_{i} / \sigma_{i}^{2}}{\sum_{i} 1 / \sigma_{i}^{2}}$,

where $d_{i}$ are the distance moduli and $\sigma_{i}$ are the absolute errors on these distance moduli. Equation (1) supposes that the relative error on distance measurements is independent of distance and that the p.d.f. of the distance moduli (proportional to $\log$ 
distance) is Gaussian. The error on the global distance modulus is (e.g. Bevington \& Robinson)

$\sigma_{\bar{d}}^{2}=\frac{1}{\sum_{i} 1 / \sigma_{i}^{2}}$.

Although this estimator of the error on the distance modulus is independent on the scatter of the measurements, we show in Appendix A (Eqs. (A.7) and (A.8)) that Eqs. (1) and (2) also represent the median and the half-width (as defined by $\left(x_{84}-\right.$ $\left.x_{16}\right) / 2$ ) of the likelihood function, respectively.

Table 2 provides our final sample of 63 early-type galaxies. Columns (1)-(3) give the name of the galaxy, with its coordinates in Cols. (4) and (5). In Col. (6), we give the distance (in Mpc, from Eq. (1)) and its relative error (Eq. (2)) in Col. (7). Column (8) gives the velocity relative to the Local Group and Col. (9) provides the sources for the distance.

Figure 1 shows a wedge diagram of the Virgo region with the 61 early-types shown as circles and the 146 spirals shown as triangles. One clearly notices the concentration of elliptical galaxies at a distance of $D \simeq 17 \mathrm{Mpc}$, which is spread out in velocity space.

\section{Observed and simulated velocity-distance relation towards the Virgo Cluster}

For a better understanding of the kinematics of galaxies in the direction of the Virgo cluster, we have simulated an observation of the velocity-distance relation using the dark matter particles in the GalICS cosmological simulation.

\subsection{N-body simulations}

The $N$-body simulations used here to find $N$-body replica of the Virgo cluster were carried out by Ninin (1999), as described by Hatton et al. (2003) in the context of the GalICS hybrid $N$-body/semi-analytic model of hierarchical galaxy formation. Here, we are only interested in the density and velocity fields directly traced by dark matter particles. The $N$-body simulation contains $256^{3}$ particles of mass $8.3 \times 10^{9} M_{\odot}$ in a box of $150 \mathrm{Mpc}$ size and it is run with a softening length amounting to a spatial resolution of $29 \mathrm{kpc}$. The simulation was run for a flat universe with cosmological parameters $\Omega_{0}=0.333, \Omega_{\Lambda}=$ $0.667, H_{0}=66.7 \mathrm{~km} \mathrm{~s}^{-1} \mathrm{Mpc}^{-1}$, and $\sigma_{8}=0.88$. Once the simulation is run, halos of dark matter are detected with a "Friendsof-Friends" (FoF) algorithm (Davis et al. 1985), with a variable linking length such that the minimum mass of the FoF groups is $1.65 \times 10^{11} M_{\odot}(20$ particles $)$ at any time step. With this method, over $2 \times 10^{4}$ halos are detected at the final timestep, corresponding to the present-day $(z=0)$ Universe. The GalICS halo finder does not allow halos within halos, so that a cluster, to which is assigned a massive halo, cannot contain smaller halos within it.

\subsection{Picking isolated halos}

Among the 12 halos that GalICS produces at $z=0$ with $M_{\mathrm{FoF}}>$ $10^{14} M_{\odot}$, we wish to choose those that resemble the Virgo cluster, both in terms of mass and environment.
As we can see in the radial phase space diagrams of dark matter halos of the simulations (Fig. 1 of Mamon et al. 2004), it is quite common to find small groups around the main cluster halo, whereas the Virgo cluster is not known to have massive neighbors, except for the M 49 group just within the virial radius. Note that the size of the simulation box, $L=150 \mathrm{Mpc}$, implies, given the periodic boundary condition, a maximum separation between halos of $\sqrt{3}(L / 2)=130 \mathrm{Mpc}$.

The effect of a neighboring halo will be from its tide on the test halo. The ratio of the tidal acceleration on a particle of the test halo at distance $r$ from its center caused by a neighboring halo at distance $R$ from the center of the first halo to the acceleration of this particle by the potential of its halo amounts to a mean density criterion:

$a_{\text {tid }} \approx \frac{G M_{2}(R)}{R^{3}} r$

and

$a_{\text {halo }}=\frac{G M_{1}(r)}{r^{2}}$

so that

$\frac{a_{\text {tid }}}{a_{\text {halo }}} \approx \frac{\rho_{2}(R)}{\rho_{1}(r)}$,

where the densities are average (and not local) and subscripts 1 and 2 stand for the central and the neighboring halo respectively. For clarity, we denote $r_{1}, M_{1}, r_{2}$ and $M_{2}$ the virial radii and masses for the test and neighboring halo, respectively (i.e. we drop the " 100 " subscript). Then, equating $r=r_{1}$, noting that the mean density at the virial radius is the same for each halo, i.e. 100 times the critical density of the Universe, and writing

$\eta=\frac{R}{r_{2}}$

the influence of each neighbor is measured by the mean density of the neighbor measured at the center of the test halo

$$
\begin{aligned}
\rho_{2}(R) & =\frac{3 M_{2}(R)}{4 \pi R^{3}} \\
& \propto \frac{M_{2}(R) / M_{2}}{\eta^{3}}=\left\{\eta^{3}[\ln \eta-\eta /(1+\eta)]\right\}^{-1},
\end{aligned}
$$

where we assumed NFW density profiles and where the last equality is from Cole \& Lacey (1996). Since the mean density is a monotonously decreasing function of radius (i.e. the function of $\eta$ in Eq. (7) decreases with $\eta$ ), the most isolated halos will have the highest minimum values of $\eta$.

The output from GalICS includes the mass of each halo obtained from the FoF estimator, so that one can easily perform a first guess of which of the halos with mass close to that of Virgo are as isolated as appears the Virgo cluster. Table 3 lists for the 10 most massive halos in the simulation, the parameters of its most perturbing neighbor (that with the lowest value of $\eta$ ) using FoF quantities for radii and masses. Columns 2 to 4 list the virial radius, mass and circular velocity obtained using the more precise spherical overdensity (SO) method, which allow a direct comparison with the values we obtained for the Virgo cluster in Mamon et al. (2004). 
Table 2. Final sample of early-type galaxies.

\begin{tabular}{|c|c|c|c|c|c|c|c|c|}
\hline \multicolumn{3}{|c|}{ Galaxy } & RA & Dec & \multirow{2}{*}{$\begin{array}{c}D \\
(\mathrm{Mpc})\end{array}$} & \multirow[t]{2}{*}{ Rel. error } & \multirow{2}{*}{$\begin{array}{c}v \\
\left(\mathrm{~km} \mathrm{~s}^{-1}\right)\end{array}$} & \multirow[t]{2}{*}{ sources } \\
\hline VCC & NGC & Messier & \multicolumn{2}{|c|}{$(\mathrm{J} 2000)$} & & & & \\
\hline 49 & 4168 & - & $12^{\mathrm{h}} 12^{\mathrm{m}} 17^{\mathrm{s}} \cdot 3$ & $+13^{\circ} 12^{\prime} 17^{\prime \prime}$ & 34.20 & 0.41 & 2128 & $\mathrm{G}$ \\
\hline 220 & 4233 & - & $12^{\mathrm{h}} 17^{\mathrm{m}} 07^{\mathrm{s}} .7$ & $+07^{\circ} 37^{\prime} 26^{\prime \prime}$ & 32.96 & 0.41 & 2181 & $\mathrm{G}$ \\
\hline 312 & 4255 & - & $12^{\mathrm{h}} 18^{\mathrm{m}} 56^{\mathrm{s}} .1$ & $+04^{\circ} 47^{\prime} 09^{\prime \prime}$ & 28.71 & 0.41 & 1603 & G \\
\hline 319 & - & - & $12^{\mathrm{h}} 19^{\mathrm{m}} 02^{\mathrm{s}} .0$ & $+13^{\circ} 58^{\prime} 48^{\prime \prime}$ & 7.59 & 0.38 & -266 & $\mathrm{Y}_{L} \mathrm{Y}_{R}$ \\
\hline 342 & 4259 & - & $12^{\mathrm{h}} 19^{\mathrm{m}} 22^{\mathrm{s}} \cdot 2$ & $+05^{\circ} 22^{\prime} 34^{\prime \prime}$ & 57.02 & 0.41 & 2306 & $\mathrm{G}$ \\
\hline 345 & 4261 & - & $12^{\mathrm{h}} 19^{\mathrm{m}} 23^{\mathrm{s}} .2$ & $+05^{\circ} 49^{\prime} 32^{\prime \prime}$ & 32.27 & 0.16 & 2012 & GT \\
\hline 355 & 4262 & - & $12^{\mathrm{h}} 19^{\mathrm{m}} 30^{\mathrm{s}} \cdot 6$ & $+14^{\circ} 52^{\prime} 38^{\prime \prime}$ & 14.86 & 0.41 & 1181 & $\mathrm{G}$ \\
\hline 369 & 4267 & - & $12^{\mathrm{h}} 19^{\mathrm{m}} 45^{\mathrm{s}} .4$ & $+12^{\circ} 47^{\prime} 53^{\prime \prime}$ & 14.66 & 0.41 & 852 & $\mathrm{G}$ \\
\hline 575 & 4318 & - & $12^{\mathrm{h}} 22^{\mathrm{m}} 43^{\mathrm{s}} \cdot 3$ & $+08^{\circ} 11^{\prime} 52^{\prime \prime}$ & 25.35 & 0.41 & 1047 & $\mathrm{G}$ \\
\hline 648 & 4339 & - & $12^{\mathrm{h}} 23^{\mathrm{m}} 34^{\mathrm{s}} .9$ & $+06^{\circ} 04^{\prime} 54^{\prime \prime}$ & 16.44 & 0.17 & 1108 & $\mathrm{~T}$ \\
\hline 685 & 4350 & - & $12^{\mathrm{h}} 23^{\mathrm{m}} 57^{\mathrm{s}} .7$ & $+16^{\circ} 41^{\prime} 33^{\prime \prime}$ & 14.86 & 0.41 & 1049 & $\mathrm{G}$ \\
\hline 731 & 4365 & - & $12^{\mathrm{h}} 24^{\mathrm{m}} 28^{\mathrm{s}} \cdot 3$ & $+07^{\circ} 19^{\prime} 04^{\prime \prime}$ & 22.07 & 0.05 & 1058 & $\mathrm{~J}_{I} \mathrm{~J}_{K} \mathrm{NT}$ \\
\hline 763 & 4374 & 84 & $12^{\mathrm{h}} 25^{\mathrm{m}} 03^{\mathrm{s}} .7$ & $+12^{\circ} 53^{\prime} 13^{\prime \prime}$ & 17.71 & 0.07 & 733 & GNT \\
\hline 778 & 4377 & - & $12^{\mathrm{h}} 25^{\mathrm{m}} 12^{\mathrm{s}} \cdot 3$ & $+14^{\circ} 45^{\prime} 43^{\prime \prime}$ & 15.49 & 0.41 & 1182 & G \\
\hline 784 & 4379 & - & $12^{\mathrm{h}} 25^{\mathrm{m}} 14^{\mathrm{s}} .8$ & $+15^{\circ} 36^{\prime} 26^{\prime \prime}$ & 14.19 & 0.38 & 871 & $\mathrm{~T}$ \\
\hline 810 & - & - & $12^{\mathrm{h}} 25^{\mathrm{m}} 33^{\mathrm{s}} .8$ & $+13^{\circ} 13^{\prime} 30^{\prime \prime}$ & 12.36 & 0.38 & -516 & $\mathrm{Y}_{L} \mathrm{Y}_{R}$ \\
\hline 828 & 4387 & - & $12^{\mathrm{h}} 25^{\mathrm{m}} 41^{\mathrm{s}} .8$ & $+12^{\circ} 48^{\prime} 35^{\prime \prime}$ & 17.21 & 0.35 & 373 & GT \\
\hline 881 & 4406 & 86 & $12^{\mathrm{h}} 26^{\mathrm{m}} 12^{\mathrm{s}} .2$ & $+12^{\circ} 56^{\prime} 44^{\prime \prime}$ & 17.40 & 0.04 & -465 & $\mathrm{~J}_{I} \mathrm{~J}_{K} \mathrm{KNT}$ \\
\hline 940 & - & - & $12^{\mathrm{h}} 26^{\mathrm{m}} 47^{\mathrm{s}} .1$ & $+12^{\circ} 27^{\prime} 14^{\prime \prime}$ & 7.24 & 0.54 & 1337 & $\mathrm{Y}_{R}$ \\
\hline 953 & - & - & $12^{\mathrm{h}} 26^{\mathrm{m}} 54^{\mathrm{s}} .7$ & $+13^{\circ} 33^{\prime} 57^{\prime \prime}$ & 18.88 & 0.38 & -678 & $\mathrm{Y}_{L} \mathrm{Y}_{R}$ \\
\hline 965 & - & - & $12^{\mathrm{h}} 27^{\mathrm{m}} 03^{\mathrm{s}} .1$ & $+12^{\circ} 33^{\prime} 39^{\prime \prime}$ & 9.29 & 0.54 & 614 & $\mathrm{Y}_{R}$ \\
\hline 1025 & 4434 & - & $12^{\mathrm{h}} 27^{\mathrm{m}} 36^{\mathrm{s}} .7$ & $+08^{\circ} 09^{\prime} 14^{\prime \prime}$ & 25.50 & 0.15 & 897 & GT \\
\hline 1030 & 4435 & - & $12^{\mathrm{h}} 27^{\mathrm{m}} 40^{\mathrm{s}} .6$ & $+13^{\circ} 04^{\prime} 44^{\prime \prime}$ & 13.12 & 0.41 & 611 & $\mathrm{G}$ \\
\hline 1146 & 4458 & - & $12^{\mathrm{h}} 28^{\mathrm{m}} 57^{\mathrm{s}} \cdot 6$ & $+13^{\circ} 14^{\prime} 29^{\prime \prime}$ & 17.97 & 0.09 & 499 & GKNT \\
\hline 1173 & - & - & $12^{\mathrm{h}} 29^{\mathrm{m}} 14^{\mathrm{s}} .8$ & $+12^{\circ} 58^{\prime} 41^{\prime \prime}$ & 14.96 & 0.38 & 2292 & $\mathrm{Y}_{L} \mathrm{Y}_{R}$ \\
\hline 1196 & 4468 & - & $12^{\mathrm{h}} 29^{\mathrm{m}} 30^{\mathrm{s}} .9$ & $+14^{\circ} 02^{\prime} 55^{\prime \prime}$ & 15.85 & 0.13 & 732 & $\mathrm{~T}$ \\
\hline 1226 & 4472 & 49 & $12^{\mathrm{h}} 29^{\mathrm{m}} 46^{\mathrm{s}} .7$ & $+07^{\circ} 59^{\prime} 59^{\prime \prime}$ & 16.27 & 0.03 & 693 & $\mathrm{GJ}_{I} \mathrm{~J}_{K} \mathrm{KNT}$ \\
\hline 1231 & 4473 & - & $12^{\mathrm{h}} 29^{\mathrm{m}} 48^{\mathrm{s}} .9$ & $+13^{\circ} 25^{\prime} 46^{\prime \prime}$ & 17.12 & 0.05 & 2041 & GKNT \\
\hline 1242 & 4474 & - & $12^{\mathrm{h}} 29^{\mathrm{m}} 53^{\mathrm{s}} .7$ & $+14^{\circ} 04^{\prime} 06^{\prime \prime}$ & 15.14 & 0.41 & 1405 & $\mathrm{G}$ \\
\hline 1250 & 4476 & - & $12^{\mathrm{h}} 29^{\mathrm{m}} 59^{\mathrm{s}} .1$ & $+12^{\circ} 20^{\prime} 55^{\prime \prime}$ & 19.08 & 0.11 & 1767 & NT \\
\hline 1253 & 4477 & - & $12^{\mathrm{h}} 30^{\mathrm{m}} 02^{\mathrm{s}} \cdot 2$ & $+13^{\circ} 38^{\prime} 11^{\prime \prime}$ & 16.44 & 0.41 & 1164 & $\mathrm{G}$ \\
\hline 1279 & 4478 & - & $12^{\mathrm{h}} 30^{\mathrm{m}} 17^{\mathrm{s}} .5$ & $+12^{\circ} 19^{\prime} 40^{\prime \prime}$ & 16.68 & 0.09 & 1220 & GNT \\
\hline 1297 & $4486 \mathrm{~B}$ & - & $12^{\mathrm{h}} 30^{\mathrm{m}} 32^{\mathrm{s}} .0$ & $+12^{\circ} 29^{\prime} 27^{\prime \prime}$ & 16.31 & 0.03 & 136 & $\mathrm{KN}$ \\
\hline 1308 & - & - & $12^{\mathrm{h}} 30^{\mathrm{m}} 45^{\mathrm{s}} .9$ & $+11^{\circ} 20^{\prime} 36^{\prime \prime}$ & 17.86 & 0.38 & 1511 & $\mathrm{Y}_{L} \mathrm{Y}_{R}$ \\
\hline 1316 & 4486 & 87 & $12^{\mathrm{h}} 30^{\mathrm{m}} 49^{\mathrm{s}} .4$ & $+12^{\circ} 23^{\prime} 28^{\prime \prime}$ & 16.82 & 0.09 & 1095 & GNT \\
\hline 1321 & 4489 & - & $12^{\mathrm{h}} 30^{\mathrm{m}} 52^{\mathrm{s}} .3$ & $+16^{\circ} 45^{\prime} 30^{\prime \prime}$ & 17.04 & 0.13 & 772 & $\mathrm{~J}_{K} \mathrm{~T}$ \\
\hline 1386 & - & - & $12^{\mathrm{h}} 31^{\mathrm{m}} 51^{\mathrm{s}} .5$ & $+12^{\circ} 39^{\prime} 24^{\prime \prime}$ & 7.08 & 0.38 & 1135 & $\mathrm{Y}_{L} \mathrm{Y}_{R}$ \\
\hline 1412 & 4503 & - & $12^{\mathrm{h}} 32^{\mathrm{m}} 06^{\mathrm{s}} .3$ & $+11^{\circ} 10^{\prime} 35^{\prime \prime}$ & 11.75 & 0.41 & 1184 & $\mathrm{G}$ \\
\hline 1420 & - & - & $12^{\mathrm{h}} 32^{\mathrm{m}} 12^{\mathrm{s}} \cdot 3$ & $+12^{\circ} 03^{\prime} 42^{\prime \prime}$ & 11.75 & 0.38 & 847 & $\mathrm{Y}_{L} \mathrm{Y}_{R}$ \\
\hline 1489 & - & - & $12^{\mathrm{h}} 33^{\mathrm{m}} 14^{\mathrm{s}} .0$ & $+10^{\circ} 55^{\prime} 43^{\prime \prime}$ & 11.30 & 0.38 & -94 & $\mathrm{Y}_{L} \mathrm{Y}_{R}$ \\
\hline 1535 & 4526 & - & $12^{\mathrm{h}} 34^{\mathrm{m}} 03.1$ & $+07^{\circ} 41^{\prime} 57^{\prime \prime}$ & 16.61 & 0.17 & 427 & GT \\
\hline 1537 & 4528 & - & $12^{\mathrm{h}} 34^{\mathrm{m}} 06^{\mathrm{s}} .2$ & $+11^{\circ} 19^{\prime} 15^{\prime \prime}$ & 14.52 & 0.41 & 1195 & $\mathrm{G}$ \\
\hline 1539 & - & - & $12^{\mathrm{h}} 34^{\mathrm{m}} 06^{\mathrm{s}} .3$ & $+12^{\circ} 44^{\prime} 40^{\prime \prime}$ & 8.83 & 0.54 & 1216 & $\mathrm{Y}_{R}$ \\
\hline 1549 & - & - & $12^{\mathrm{h}} 34^{\mathrm{m}} 14^{\mathrm{s}} .9$ & $+11^{\circ} 04^{\prime} 16^{\prime \prime}$ & 19.32 & 0.54 & 1202 & $\mathrm{Y}_{R}$ \\
\hline 1619 & 4550 & - & $12^{\mathrm{h}} 35^{\mathrm{m}} 30^{\mathrm{s}} .7$ & $+12^{\circ} 13^{\prime} 13^{\prime \prime}$ & 17.84 & 0.09 & 208 & KNT \\
\hline 1630 & 4551 & - & $12^{\mathrm{h}} 35^{\mathrm{m}} 38^{\mathrm{s}} .1$ & $+12^{\circ} 15^{\prime} 49^{\prime \prime}$ & 17.24 & 0.15 & 973 & GT \\
\hline 1632 & 4552 & 89 & $12^{\mathrm{h}} 35^{\mathrm{m}} 40^{\mathrm{s}} .0$ & $+12^{\circ} 33^{\prime} 22^{\prime \prime}$ & 15.31 & 0.04 & 115 & $\mathrm{GJ}_{I} \mathrm{~J}_{K} \mathrm{KNT}$ \\
\hline 1664 & 4564 & - & $12^{\mathrm{h}} 36^{\mathrm{m}} 27^{\mathrm{s}} .0$ & $+11^{\circ} 26^{\prime} 18^{\prime \prime}$ & 14.86 & 0.15 & 947 & GT \\
\hline 1669 & - & - & $12^{\mathrm{h}} 36^{\mathrm{m}} 30^{\mathrm{s}} \cdot 6$ & $+13^{\circ} 38^{\prime} 18^{\prime \prime}$ & 8.79 & 0.54 & 427 & $\mathrm{Y}_{R}$ \\
\hline 1692 & 4570 & - & $12^{\mathrm{h}} 36^{\mathrm{m}} 53^{\mathrm{s}} .5$ & $+07^{\circ} 14^{\prime} 46^{\prime \prime}$ & 13.80 & 0.41 & 1559 & $\mathrm{G}$ \\
\hline 1720 & 4578 & - & $12^{\mathrm{h}} 37^{\mathrm{m}} 30^{\mathrm{s}} .6$ & $+09^{\circ} 33^{\prime} 16^{\prime \prime}$ & 17.94 & 0.06 & 2102 & $\mathrm{GJ}_{I} \mathrm{~J}_{K} \mathrm{~T}$ \\
\hline 1834 & 4600 & - & $12^{\mathrm{h}} 40^{\mathrm{m}} 23^{\mathrm{s}} .0$ & $+03^{\circ} 07^{\prime} 02^{\prime \prime}$ & 7.35 & 0.20 & 615 & $\mathrm{~T}$ \\
\hline 1869 & 4608 & - & $12^{\mathrm{h}} 41^{\mathrm{m}} 13^{\mathrm{s}} \cdot 4$ & $+10^{\circ} 09^{\prime} 18^{\prime \prime}$ & 20.23 & 0.41 & 1633 & G \\
\hline 1883 & 4612 & - & $12^{\mathrm{h}} 41^{\mathrm{m}} 32^{\mathrm{s}} .8$ & $+07^{\circ} 18^{\prime} 50^{\prime \prime}$ & 14.06 & 0.41 & 1697 & $\mathrm{G}$ \\
\hline 1902 & 4620 & - & $12^{\mathrm{h}} 41^{\mathrm{m}} 59.4$ & $+12^{\circ} 56^{\prime} 32^{\prime \prime}$ & 21.28 & 0.28 & 1012 & $\mathrm{~T}$ \\
\hline 1903 & 4621 & 59 & $12^{\mathrm{h}} 42^{\mathrm{m}} 02^{\mathrm{s}} \cdot 3$ & $+11^{\circ} 38^{\prime} 45^{\prime \prime}$ & 14.80 & 0.05 & 258 & GKNT \\
\hline
\end{tabular}


Table 2. continued.

\begin{tabular}{|c|c|c|c|c|c|c|c|c|}
\hline \multicolumn{3}{|c|}{ Galaxy } & RA & Dec & \multirow{2}{*}{$\begin{array}{c}D \\
(\mathrm{Mpc})\end{array}$} & Rel. error & \multirow{2}{*}{$\begin{array}{c}v \\
\left(\mathrm{~km} \mathrm{~s}^{-1}\right)\end{array}$} & \multirow{2}{*}{ sources } \\
\hline$\overline{\mathrm{VCC}}$ & NGC & Messier & \multicolumn{2}{|c|}{$(\mathrm{J} 2000)$} & & & & \\
\hline 1938 & 4638 & - & $12^{\mathrm{h}} 42^{\mathrm{m}} 47^{\mathrm{s}} .5$ & $+11^{\circ} 26^{\prime} 32^{\prime \prime}$ & 17.33 & 0.21 & 956 & GT \\
\hline 1939 & 4624 & - & $12^{\mathrm{h}} 42^{\mathrm{m}} 50^{\mathrm{s}} \cdot 0$ & $+02^{\circ} 41^{\prime} 16^{\prime \prime}$ & 15.14 & 0.06 & 924 & $\mathrm{GJ}_{I} \mathrm{~J}_{K} \mathrm{~T}$ \\
\hline 1978 & 4649 & 60 & $12^{\mathrm{h}} 43^{\mathrm{m}} 40{ }^{\mathrm{s}} .1$ & $+11^{\circ} 33^{\prime} 08^{\prime \prime}$ & 16.36 & 0.04 & 969 & GKNT \\
\hline 2000 & 4660 & - & $12^{\mathrm{h}} 44^{\mathrm{m}} 32^{\mathrm{s}} .0$ & $+11^{\circ} 11^{\prime} 24^{\prime \prime}$ & 15.66 & 0.11 & 914 & GNT \\
\hline 2087 & 4733 & - & $12^{\mathrm{h}} 51^{\mathrm{m}} 06^{\mathrm{s}} .8$ & $+10^{\circ} 54^{\prime} 43^{\prime \prime}$ & 14.93 & 0.18 & 772 & $\mathrm{~T}$ \\
\hline 2092 & 4754 & - & $12^{\mathrm{h}} 52^{\mathrm{m}} 17^{\mathrm{s}} .5$ & $+11^{\circ} 18^{\prime} 50^{\prime \prime}$ & 16.94 & 0.12 & 1206 & GT \\
\hline 2095 & 4762 & - & $12^{\mathrm{h}} 52^{\mathrm{m}} 55^{\mathrm{s}} .9$ & $+11^{\circ} 13^{\prime} 49^{\prime \prime}$ & 10.81 & 0.41 & 818 & $\mathrm{G}$ \\
\hline
\end{tabular}

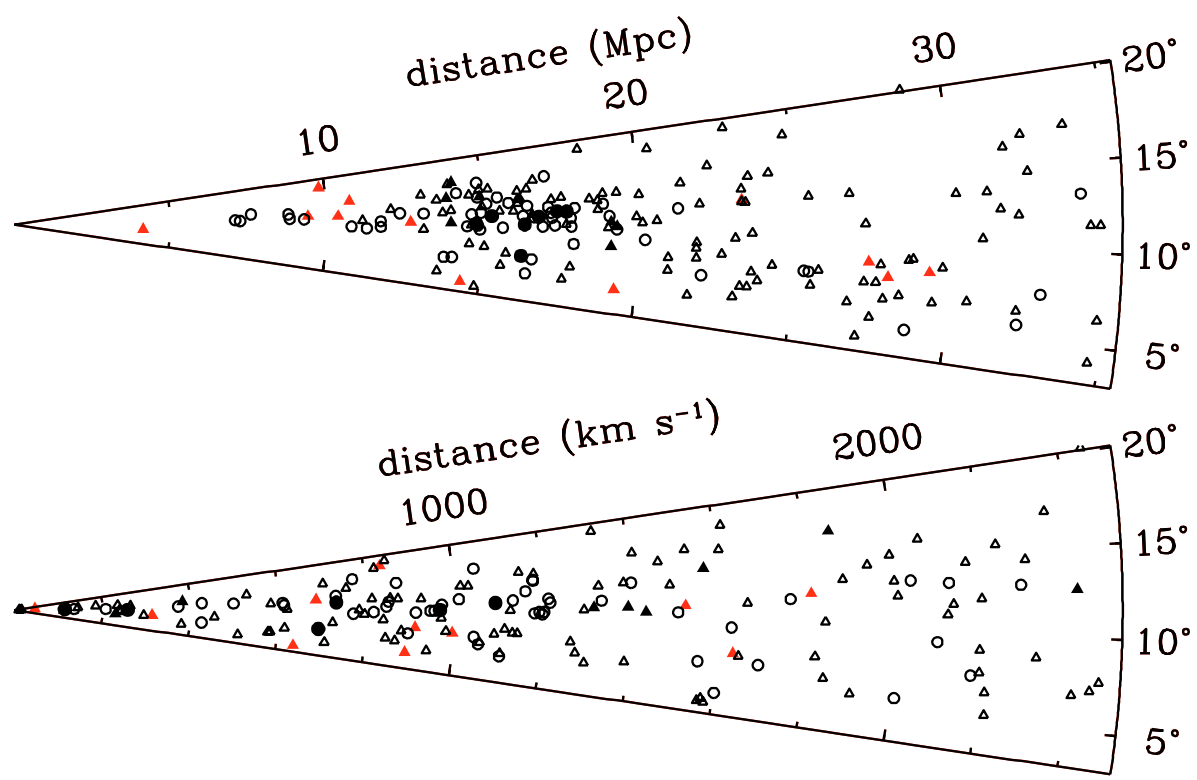

Fig. 1. Positions of 146 spiral (triangles) and 61 elliptical (circles) galaxies in the Virgo field (within $9^{\circ}$ from M 87) as a function of Declination and distance (assumed correct, top) and radial velocity (bottom). H I-deficient galaxies (deviating more than $3 \sigma$ from normalcy) are shown as filled triangles - with the ones apparently outside the cluster (Table 7, below) shown as surrounded filled triangles (red triangles in the electronic version of the Journal), while Messier ellipticals (see Table 2) are shown as filled circles, with M49 to the South, and M 59, M 89, M 60, M 87, M 86 and M 84 at increasing distances (top plot) and M 89, M 59, M 84, M 60 and M 87 at increasing radial velocities (bottom plot, M 86 has $v<0)$.

Table 3. Most perturbing neighbors of 10 most massive halos in GalICS simulation.

\begin{tabular}{ccccccc}
\hline \hline $\begin{array}{c}\text { rank } \\
(\mathrm{FoF})\end{array}$ & $\begin{array}{c}r_{1}^{\mathrm{SO}} \\
(\mathrm{Mpc})\end{array}$ & $\begin{array}{c}M_{1}^{\mathrm{SO}} \\
\left(10^{14} M_{\odot}\right)\end{array}$ & $\begin{array}{c}v_{1}^{\mathrm{SO}} \\
\left(\mathrm{km} \mathrm{s}^{-1}\right)\end{array}$ & $\frac{R}{r_{1}^{\mathrm{SO}}}$ & $\frac{M_{2}^{\mathrm{FoF}}}{M_{1}^{\mathrm{FoF}}}$ & $\eta_{\min }$ \\
\hline 1 & 3.19 & 16.7 & 1503 & 0.55 & 0.011 & 3.7 \\
2 & 2.42 & 7.4 & 1143 & 0.58 & 0.003 & 3.1 \\
3 & 2.50 & 8.1 & 1180 & 0.55 & 0.020 & 2.4 \\
4 & 2.54 & 8.5 & 1200 & 0.53 & 0.011 & 2.4 \\
5 & 2.10 & 4.8 & 989 & 0.62 & 0.001 & 2.9 \\
6 & 2.00 & 4.1 & 942 & 0.58 & 0.001 & 2.5 \\
7 & 1.97 & 3.9 & 927 & 0.57 & 0.031 & 3.4 \\
8 & 1.78 & 2.9 & 842 & 0.61 & 0.002 & 2.5 \\
9 & 1.84 & 3.2 & 869 & 0.58 & 0.103 & 2.2 \\
10 & 2.03 & 4.3 & 958 & 0.51 & 0.003 & 2.6 \\
\hline
\end{tabular}

As seen in Table 3, the most perturbing halo is always inside $r_{1}$. Therefore, it is not clear that we can easily estimate the exact virial radius and mass of each perturbing halo with the same spherical overdensity method as in Mamon et al. (2004) to produce a better estimate of isolated halos. As we expect $r_{1}^{\text {SO }}$ to be approximately proportional to $r_{1}^{\mathrm{FoF}}$, we take the results of Table 3 as good indicators of the degree of isolation of halos in the simulations.

As shown in Table 3, the most isolated halo in the simulations is halo 1 , the most massive halo. Picking the most massive halo also ensures that no other massive halos will distort the mock velocity-distance relation.

\subsection{Rescaling the simulations to the scales of the Virgo cluster}

We now need to rescale the simulated clusters to Virgo. A reasonable way to put two halos in comparable units is to normalize distances with the virial radius, $r_{100}$, and velocities with the circular velocity of the halo at the virial radius, $v_{100}$. We therefore convert from the simulation frame to the Virgo cluster frame by rescaling the terms related to distance with the 
factor (omitting the "100" subscripts for clarity) $r_{\mathrm{V}} / r_{\mathrm{s}}$ and the terms related to velocities with the analogous factor $v_{\mathrm{V}} / v_{\mathrm{s}}$, where superscripts "s" and "V" refer to the simulation and the Virgo cluster, respectively. We adopt the Virgo cluster virial radius and circular velocity derived by Mamon et al. (2004) from X-ray observations $r_{\mathrm{V}}=1.65 h_{2 / 3}^{-1} \mathrm{Mpc}$, where $h_{2 / 3}=$ $H_{0} /\left(66.7 \mathrm{~km} \mathrm{~s}^{-1} \mathrm{Mpc}^{-1}\right)$, and $v_{\mathrm{V}}=780 \mathrm{~km} \mathrm{~s}^{-1}$.

Moreover, the $N$-body simulations give us comoving velocities $\boldsymbol{u}_{\mathrm{s}}$ of dark matter particles, to which we must add the Hubble flow. Therefore:

$$
\begin{aligned}
D & =D_{\mathrm{s}} \frac{r_{\mathrm{V}}}{r_{\mathrm{s}}} \\
v & =u_{\mathrm{s}} \frac{v_{\mathrm{V}}}{v_{\mathrm{s}}}+H_{0} D
\end{aligned}
$$

where $u_{\mathrm{s}}$ represents the comoving radial velocity of the particle in the simulation with respect to the observer in comoving units.

Inverting Eq. (8), we need to place the observer at a distance

$$
D_{\mathrm{obs}}=D_{\mathrm{V}} \frac{r_{\mathrm{s}}}{r_{\mathrm{V}}}
$$

to the halo used to mimic the Virgo cluster (in simulation units). Once we have $D_{\text {obs }}$, we can select different observers by choosing different positions on a sphere of radius $D_{\text {obs }}$ centered on the halo and assigning to each position, the mean velocity of the 10 nearest halos to the observer, so as to give the observer an appropriate peculiar velocity. As our galaxies in the Virgo cluster extend up to $D_{\max }=50 \mathrm{Mpc}$, we will need to use particles around the chosen dark matter halo up to a distance from the cluster of $D_{\max }-D_{\mathrm{V}} \simeq 20 r_{100}$.

It is interesting to check the peculiar velocities (or equivalently the systemic velocities) found for the simulated observer relative to the simulated cluster and compare them with that of our Local Group of galaxies relative to the Virgo cluster, after rescaling the simulation radial and velocity separations to the Virgo cluster attributes as in Eqs. (8) and (9). For 500 observers placed at a given distance from the halo, but in different random directions, we find a systemic velocity between the halo and the observer (i.e. the mean of 10 nearest halos) in Virgo units of $998 \pm 118 \mathrm{~km} \mathrm{~s}^{-1}$ (errors are the $1 \sigma$ dispersion) when we assume $D_{\mathrm{V}}=16.8 \mathrm{Mpc}$ (as suggested by the positions of the early-type galaxies in Fig. 1, and especially of M 87, which lies at the center of the X-ray emission) and $1308 \pm 155 \mathrm{~km} \mathrm{~s}^{-1}$ when we assume $D_{\mathrm{V}}=21 \mathrm{Mpc}$, as in Sanchis et al. (2002). The values of the systemic velocity of the Virgo cluster in the literature range approximately between 900 and $1000 \mathrm{~km} \mathrm{~s}^{-1}$ (Teerikorpi et al. 1992). Therefore, putting the Virgo cluster at $16.8 \mathrm{Mpc}$, as indicated by the early type galaxies, gives a better match to the systemic velocity of the cluster. In addition, if we calculate the number of all galaxies (early-type and late-type ones) per distance bin, we found the maximum of the distribution between 16-18 Mpc. Even if our sample lacks of completeness, this result, together with the previous ones, lead us to choose $D_{\mathrm{V}}=16.8 \mathrm{Mpc}$ for the comparison between the $N$-body simulations and the Virgo cluster.

\subsection{Velocity-distance relation without distance errors}

We take all the objects lying within a cone of half-opening $9^{\circ}$ aligned with the axis connecting the observer to the center of the halo.

Because observed galaxy catalogs, such as the VCC (Binggeli et al. 1985) catalog of Virgo cluster galaxies, are magnitude-limited, we imposed upon our simulated catalog (of dark matter particles) the same magnitude limit as the VCC, namely $B<18$ (Binggeli et al. 1985). For this, we consider bins of distance to the observer, and, in each distance bin, we remove a fraction of dark matter particles equal to the predicted fraction of particles fainter than $B=18$ given the distance and the Gaussian-shaped luminosity function of spiral galaxies derived by Sandage et al. (1985). This method supposes that the luminosity function is independent of environment (i.e. distance to the center of the Virgo cluster) and that those VCC galaxies with redshift-independent distance measurements have the same magnitude limit as the VCC galaxies in general. This luminosity incompleteness is only noticeable at large distances, where it amounts to reducing the number of particles by less than $10 \%$.

Figure 2 shows the velocity-distance plot of the simulated dark matter particles (small points), rescaled to Virgo units, and from which particles statistically fainter than the magnitude limit of the observed catalogs were removed. Superposed are the spiral galaxies (triangles) from Solanes et al. (2002) and the early-type galaxies (circles) compiled in Table 2 . We have added to our sample the spiral galaxies compiled by Solanes et al. (2002) with reliable Tully-Fisher (Tully \& Fisher 1977, hereafter TF) distances but no HI data. We list these galaxies in Table 4, with their coordinates, morphological type (from LEDA), systemic velocity and the final distance with errors assigned to it by Solanes et al. (2002), who estimated errors in distance as the mean deviation between the rescaled mean distance and the different estimates used (with this method, galaxies with only one estimator cannot be assigned an error and their distances are considered uncertain). We represent these objects as squares in Fig. 2.

Although Fig. 2 concerns a single observer and a single halo to represent the Virgo cluster (i.e. halo 1, which is the most isolated), the results are very similar for all halos studied as well as for any observer whose velocity with respect to the center of the main halo is similar to the mean of the 500 random trials. In other words, Fig. 2 provides a realistic general velocity-distance relation for a Virgo-like cluster in a flat Universe with a cosmological constant. Very recently, Klypin et al. (2003) have produced a similar plot for dark matter particles from their adaptive mesh refinement simulation. Note that the groups visible in the 3D phase space plot (Fig. 1 in Mamon et al. 2004) are much less visible here and the projection places them at smaller distances from the center of the main cluster.

Figure 2 indicates that the velocity field drawn by the observed spirals is noticeably different from the one followed by the dark matter particles of the simulation. Nevertheless, the figure shows that 1) the center of the halo corresponds to the location of greater galaxy density (not only for the early-type 


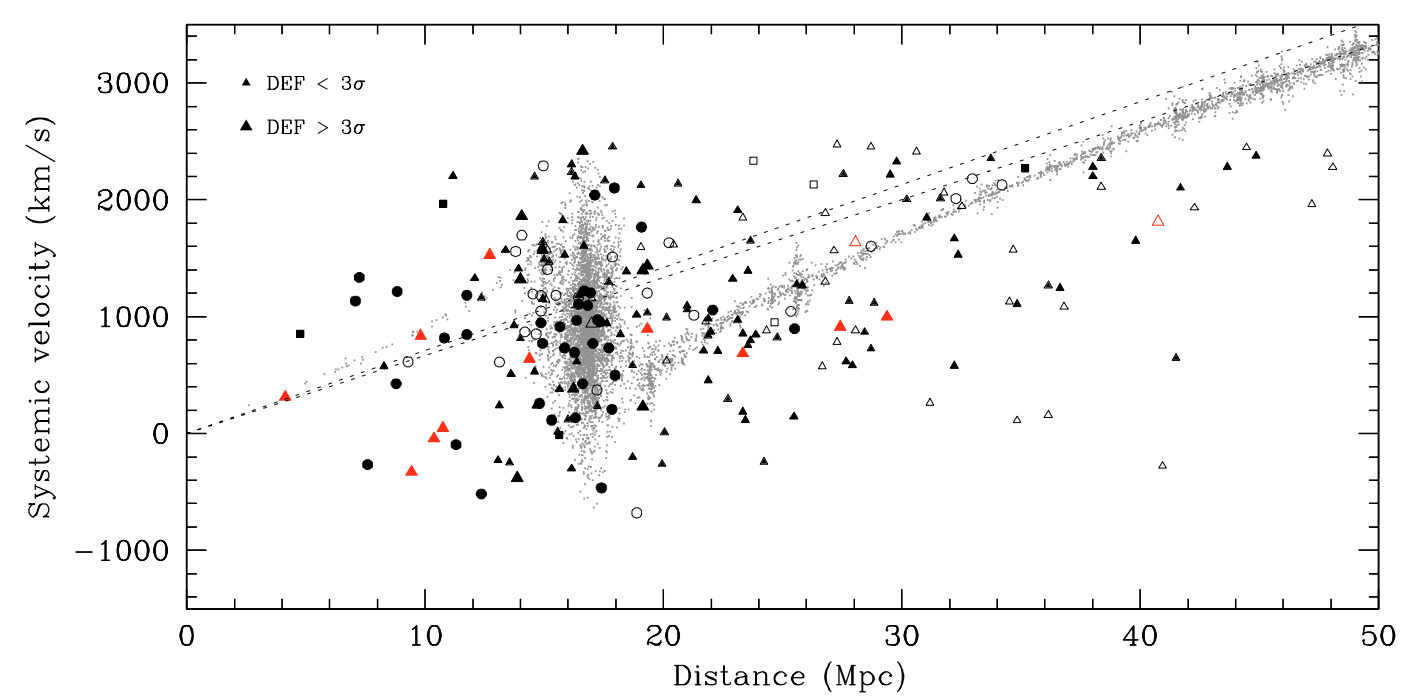

Fig. 2. Simulated and observed velocity-distance diagrams. The observer is at $(0,0)$ and sees a cone of angular radius $9^{\circ}$. Dots represent the velocity field traced by the particles ( 1 in 50 for clarity) in the cosmological $N$-body simulation in the direction of the most massive halo, for which distances and velocities have been rescaled (Eqs. (8) and (9)) incorporating a Hubble flow with $H_{0}=66.7 \mathrm{~km} \mathrm{~s}^{-1} \mathrm{Mpc}^{-1}$, as in the simulation. Superposed are galaxies of the Virgo cluster. Circles and triangles represent early-type and late-type galaxies, respectively. The size of the triangles informs about the H I-deficiency of the spiral galaxies measured in units of the mean standard deviation for field objects (=0.24). $\mathrm{H}$ I-deficient galaxies deviating more than $3 \sigma$ from normalcy apparently outside the cluster (Table 7, below) are shown as surrounded filled triangles (red triangles in the electronic version of the Journal). Squares represent additional spiral galaxies with no H I-deficiency data. Open symbols indicate galaxies with uncertain distances (with distance errors greater than $5 \mathrm{Mpc}$ or spirals with only one distance estimate). Dashed lines show the unperturbed Hubble flow with $H_{0}=66.7$ and $70 \mathrm{~km} \mathrm{~s}^{-1} \mathrm{Mpc}^{-1}$, respectively (going upwards). The locations of the vertical spikes of simulated particles away from the Virgo cluster (assumed to lie at $16.8 \mathrm{Mpc}$ ) are not meaningful and should not be compared to the measured galaxy distances.

Table 4. Spiral galaxies without H I data.

\begin{tabular}{|c|c|c|c|c|c|c|}
\hline \multicolumn{2}{|c|}{ Galaxy } & RA & Dec & $T$ & $v$ & $D$ \\
\hline VCC & NGC & \multicolumn{2}{|c|}{ (J2000) } & & $\left(\mathrm{km} \mathrm{s}^{-1}\right)$ & $(\mathrm{Mpc})$ \\
\hline 132 & - & $12^{\mathrm{h}} 15^{\mathrm{m}} 03^{\mathrm{s}} .9$ & $+13^{\circ} 01^{\prime} 55^{\prime \prime}$ & 8 & 1965 & $10.76_{-2.10}^{+2.60}$ \\
\hline 343 & - & $12^{\mathrm{h}} 19^{\mathrm{m}} 22^{\mathrm{s}} .0$ & $+07^{\circ} 52^{\prime} 16^{\prime \prime}$ & 8 & 2336 & 23.77 \\
\hline 912 & 4413 & $12^{\mathrm{h}} 26^{\mathrm{m}} 32^{\mathrm{s}} .2$ & $+12^{\circ} 36^{\prime} 39^{\prime \prime}$ & 2 & -13 & $15.63_{-2.20}^{+2.57}$ \\
\hline 952 & - & $12^{\mathrm{h}} 26^{\mathrm{m}} 55^{\mathrm{s}} .5$ & $+09^{\circ} 52^{\prime} 58^{\prime \prime}$ & 5 & 853 & $4.77_{-3.88}^{+4.60}$ \\
\hline 1605 & - & $12^{\mathrm{h}} 35^{\mathrm{m}} 14^{\mathrm{s}} .5$ & $+10^{\circ} 25^{\prime} 53^{\prime \prime}$ & 5 & 951 & $24.66_{-5.34}^{+6.82}$ \\
\hline 1673 & 4567 & $12^{\mathrm{h}} 36^{\mathrm{m}} 32^{\mathrm{s}} .9$ & $+11^{\circ} 15^{\prime} 28^{\prime \prime}$ & 4 & 2133 & 26.30 \\
\hline 1933 & - & $12^{\mathrm{h}} 42^{\mathrm{m}} 44^{\mathrm{s}} .7$ & $+07^{\circ} 20^{\prime} 16^{\prime \prime}$ & 2 & 2271 & $35.16_{-0.32}^{+0.33}$ \\
\hline
\end{tabular}

galaxies used to mark the center of the Virgo cluster); 2) the thickness of the halo in velocity space (the velocity dispersion) corresponds to the corresponding thickness of the central observed galaxies, and 3) the majority of the elliptical galaxies outside the cluster also appear to fit well the location of the particles in the outer infalling/expanding locus. These 3 facts suggest that both the distance to the halo and the rescaling factors are well chosen and that the differences between the Virgo cluster and the halo in the simulation should be attributable to errors arising from the distance estimates (as the velocity errors are negligible). It appears unlikely that the galaxies that, for a given distance, lie within more than $1000 \mathrm{~km} \mathrm{~s}^{-1}$ from the locus of dark matter particles belong to the low- or high-velocity tails of large velocity dispersion groups near the cluster, since in our simulations, there are no such high velocity dispersion groups outside the main cluster.

\subsection{Velocity-distance relation with distance errors}

Given that most of the galaxies outside the velocity field drawn by dark matter particles are spirals, we now build a new velocity-distance diagram of our simulated particles incorporating the mean relative distance error for the spiral galaxy sample. Solanes et al. (2002) combined different studies of the TF relation to calculate the distances of spirals in the Virgo cluster. These studies give dispersions of the TF relation of roughly $0.4 \mathrm{mag}$, corresponding to an uncertainty of $18 \%$ in relative distance. Figure 3 shows the corresponding 


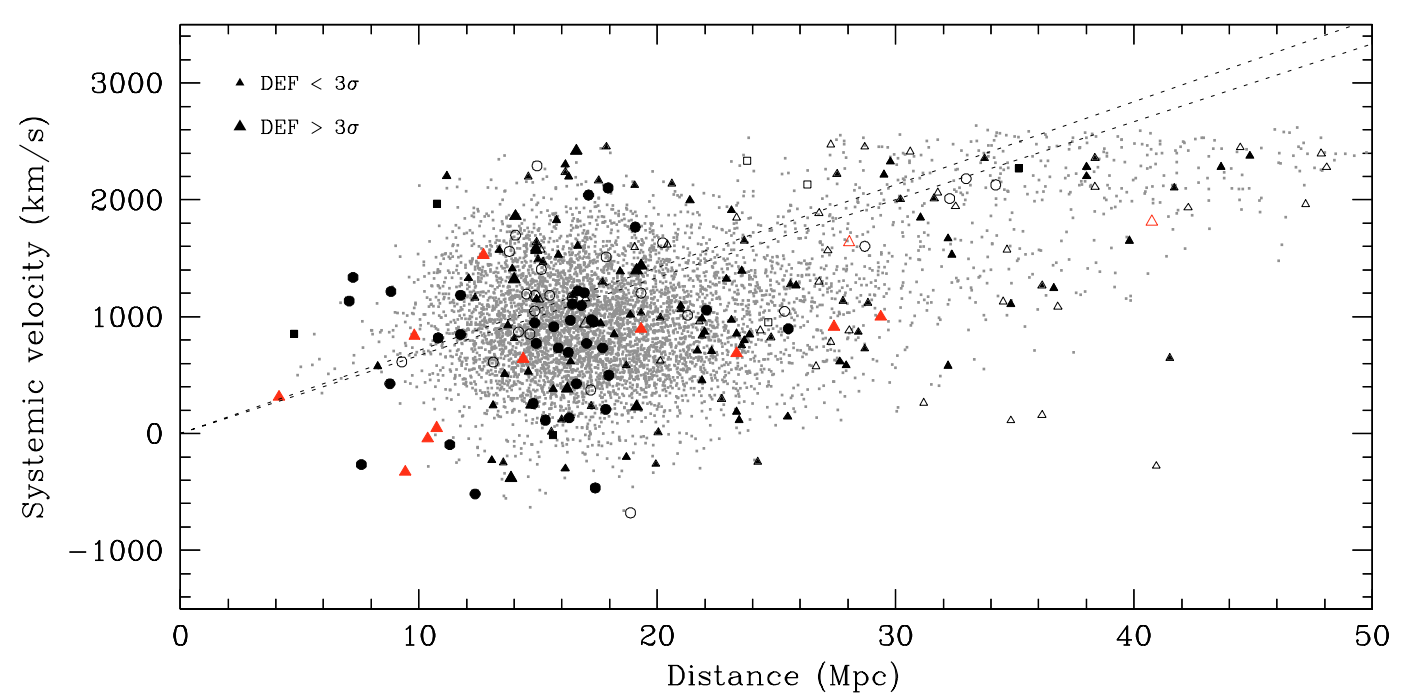

Fig. 3. Same as Fig. 2, but incorporating Gaussian relative distance errors of $\sigma \ln D=0.2$ for the dark matter particles.

Table 5. Grossly incorrect galaxy distances.

\begin{tabular}{|c|c|c|c|c|c|c|}
\hline \multicolumn{2}{|c|}{ Galaxy } & RA & Dec & \multirow[t]{2}{*}{$T$} & \multirow{2}{*}{$\begin{array}{c}v \\
\left(\mathrm{~km} \mathrm{~s}^{-1}\right)\end{array}$} & \multirow{2}{*}{$\begin{array}{r}D \\
\mathrm{Mpc}\end{array}$} \\
\hline VCC & NGC & \multicolumn{2}{|c|}{ (J2000) } & & & \\
\hline 213 & - & $12^{\mathrm{h}} 16^{\mathrm{m}} 56^{\mathrm{s}} .0$ & $+13^{\circ} 37^{\prime} 33^{\prime \prime}$ & 5 & -278 & 40.9 \\
\hline 319 & - & $12^{\mathrm{h}} 19^{\mathrm{m}} 01^{\mathrm{s}} .7$ & $+13^{\circ} 58^{\prime} 56^{\prime \prime}$ & -4 & -256 & 7.6 \\
\hline 491 & 4299 & $12^{\mathrm{h}} 21^{\mathrm{m}} 40^{\mathrm{s}} \cdot 9$ & $+11^{\circ} 30^{\prime} 03^{\prime \prime}$ & 8 & 112 & 34.8 \\
\hline 1524 & 4523 & $12^{\mathrm{h}} 33^{\mathrm{m}} 48^{\mathrm{s}} .0$ & $+15^{\circ} 10^{\prime} 05^{\prime \prime}$ & 9 & 158 & 36.1 \\
\hline 1644 & & $12^{\mathrm{h}} 35^{\mathrm{m}} 51^{\mathrm{s}} .8$ & $+13^{\circ} 51^{\prime} 33^{\prime \prime}$ & 9 & 646 & 41.5 \\
\hline 1690 & 4569 & $12^{\mathrm{h}} 36^{\mathrm{m}} 49^{\mathrm{s}} .8$ & $+13^{\circ} 09^{\prime} 48^{\prime \prime}$ & 2 & -328 & 9.4 \\
\hline
\end{tabular}

VCC $1690=$ NGC $4569=$ M $90=$ Arp 76.

velocity-distance plot for our simulated Virgo line of sight, with inclusion of Gaussian relative distance errors with $\sigma \ln D=$ 0.20 (a slightly more conservative value than 0.18 ). We omit particles beyond $40 \mathrm{Mpc}$, which corresponds to the distance (before folding in the distance errors) where the particles have a systemic velocity approximately equal to the maximum of the observed galaxy sample.

Figure 3 indicates that the inclusion of distance errors, at the $20 \%$ relative rms level, allows us to reproduce fairly well the observed velocity-distance diagram. There are still several galaxies that lie far outside the general locus of the dark matter particles. Among them are two galaxies (VCC 319, which is part of a galaxy pair and M90 = NGC $4569=$ Arp 76, which is tidally perturbed by a close neighbor) apparently in the foreground of the cluster between 7 and $10 \mathrm{Mpc}$ and with $v<$ $-200 \mathrm{~km} \mathrm{~s}^{-1}$, and four galaxies (IC 3094, NGC 4299, which is part of a pair, NGC 4253 and VCC 1644) in the background between 34 and $42 \mathrm{Mpc}$ and $v<800 \mathrm{~km} \mathrm{~s}^{-1}$. Inspection of Fig. 2 indicates that these four galaxies are at more than $2000 \mathrm{~km} \mathrm{~s}^{-1}$ from the expected locus of the Tolman-Bondi pattern given negligible distance errors. It is highly unlikely that the peculiar velocities in interacting pairs can reach values as high as $2000 \mathrm{~km} \mathrm{~s}^{-1}$, hence the distances to these galaxies appear to be grossly incorrect.
We list the most discrepant galaxies in Table 5. Most of these galaxies have a single distance measurement (the elliptical VCC 319 has a single Sérsic fit distance estimator, which is known to be inaccurate - see Table 1). However, it is a surprise to find a bright object such as NGC 4569 (M 90) in this list, especially since it has 6 different Tully-Fisher distance measurements, 5 of which yield a distance between 7.9 and $10.2 \mathrm{Mpc}$ and one (Gavazzi et al. 1999) giving $D=16.1 \mathrm{Mpc}$, which is what we expect from Fig. 2.

Since the $20 \%$ relative errors correctly reproduce the envelope of particles from the cosmological simulation, increasing the relative rms error to say $30 \%$ will not explain the distances to these discrepant spirals. We therefore conclude that the distances of the galaxies listed in Table 5 must be highly inaccurate.

In their dynamical, Tolman-Bondi, modeling of the Virgo region, Sanchis et al. (2002) only used those spiral galaxies believed to trace the outer envelope of galaxies expanding out of the Virgo cluster. Figure 2 clearly indicates that the galaxies used by Sanchis et al. (roughly aligned between distances of 34 and $45 \mathrm{Mpc}$ ) lie at typically $600 \mathrm{~km} \mathrm{~s}^{-1}$ lower velocities for their distance than predicted by our simulated velocity-distance model. Figure 3 suggests that the alignment of these galaxies in the velocity-distance plot may be fortuitous, given the $\sim 20 \%$ relative distance errors. This fortuitous alignment forced the model of Sanchis et al. to shift the center of the Virgo cluster to greater distances.

\subsection{Tolman-Bondi estimates of the Virgo cluster mass and distance}

We now use the cosmological $N$-body simulations to quantify the errors on the distance and mass to the Virgo cluster obtained by fitting a Tolman-Bondi model to the velocitydistance data outside the cluster core. We construct simulated pseudo-galaxy samples by randomly selecting as many points from the cosmological $N$-body simulation as there are galaxies in the Virgo cluster sample. We apply fixed relative distance 
Table 6. Best fitting parameters for each dataset.

\begin{tabular}{rrrrrrr}
\hline \hline Error & \multicolumn{2}{c}{$D_{\mathrm{V}}$} & & \multicolumn{2}{c}{$\log \left(M_{\mathrm{V}}\right)$} & $t_{0}$ \\
& \multicolumn{2}{c}{$(\mathrm{Mpc})$} & & \multicolumn{2}{c}{$\left(M_{\odot}\right)$} & $(\mathrm{Gyr})$ \\
\cline { 2 - 3 } & mean & $\sigma$ & & mean & $\sigma$ & \\
\hline $0 \%$ & 16.7 & 0.5 & & 14.5 & 0.2 & 14.1 \\
$5 \%$ & 16.8 & 0.5 & & 14.6 & 0.3 & 13.9 \\
$10 \%$ & 16.9 & 0.6 & & 14.9 & 0.4 & 12.8 \\
$15 \%$ & 17.0 & 0.8 & & 15.1 & 0.5 & 12.1 \\
$20 \%$ & 17.1 & 0.9 & & 15.5 & 0.5 & 10.1 \\
$25 \%$ & 17.1 & 1.0 & & 15.7 & 0.4 & 9.0 \\
$30 \%$ & 17.1 & 1.0 & & 16.1 & 0.5 & 6.7 \\
\hline
\end{tabular}

errors in the range $0 \%-30 \%$ and, for each relative distance error, we construct 50 realizations of the Virgo sample. We identify pseudo-galaxies presumably belonging to the core by building histograms of the number of pseudo-galaxies per $2 \mathrm{Mpc}$ distance bin. Such histograms peak at the core of the halo, in a region of width $\sim 4 \mathrm{Mpc}$ for the no error set and up to $\sim 12 \mathrm{Mpc}$ for the $30 \%$ error set. We avoid the virialized core by only considering pseudo-galaxies lying on the back side of the identified cluster core.

In the Tolman-Bondi model used by Sanchis et al., galaxies are treated as test particles moving in a point-mass gravitational potential that is independent of time. The model velocitydistance curve is a function of the age of the Universe, $t_{0}$, the distance to the cluster, $D_{\mathrm{V}}$, the point mass of the cluster, $M_{\mathrm{V}}$, and the systemic velocity of the cluster relative to the Local Group of galaxies, $v_{\mathrm{V}}$. Only three of these four parameters are independent, but given the poor current velocity-distance data, it is better to use only two free parameters and fix the third one. Following Sanchis et al., we have fixed $v_{\mathrm{V}}$ to the mean velocity of the main halo for the 50 realizations $\left(998 \mathrm{~km} \mathrm{~s}^{-1}\right.$ for a distance of $16.8 \mathrm{Mpc}$, see Sect. 3) and we have allowed $M_{\mathrm{V}}$ and $D_{\mathrm{V}}$ to vary freely. The resulting $t_{0}$ will help us to see the viability of the results.

Table 6 shows the results of the fits to the point-mass Tolman-Bondi relation (see Sanchis et al. 2002) for the different sets. For zero distance errors, the model reproduces fairly well the input data of $D_{\mathrm{V}}=16.8 \mathrm{Mpc}$ and $M_{\mathrm{V}}=$ $10^{14.35} M_{\odot}$ (Mamon et al. 2004), as well as the age of the Universe: $t_{0}=13.7 \mathrm{Gyr}$ from the GalICS cosmological simulations (which happens to perfectly match the age recently obtained by Spergel et al. 2003 from the WMAP CMB experiment). The table also clearly indicates that the errors in distance contribute to a slightly larger distance to the cluster (by up to $2 \%$ ), combined with a much larger mass for the cluster (up to a factor 40!). This dramatic rise in the mass decreases the resulting $t_{0}$ to unacceptably low values. Therefore, distance errors can cause the fitting of Tolman-Bondi solutions to the velocity vs. distance of galaxies to yield incorrect cluster parameters.

For the case of real data on the Virgo cluster, the same fitting procedure leads to $M_{\mathrm{V}} \sim 3 \times 10^{16} M_{\odot}$ and $D_{\mathrm{V}} \sim 17.4 \mathrm{Mpc}$, which imply $t_{0}=5.2$ Gyr. Note that the present fitting procedure takes into account all galaxies beyond the identified core of the cluster, whereas Sanchis et al. only used those galaxies that are aligned between distances of 34 and $45 \mathrm{Mpc}$ in the velocity-distance plot, deducing a very different distance to the Virgo cluster (between 20 and $21 \mathrm{Mpc}$ ). The comparison of the values of $t_{0}$ from the real data and from the simulated data convolved with different relative distance errors, suggests that the TF distances may have up to $30 \%$ relative errors.

As shown above, the estimation of the distance to the cluster provided by the point mass model is relatively good even for appreciable errors in relative distances. Therefore, one could still obtain a reasonable value for the mass of the cluster by taking $D_{\mathrm{V}}$ from the fit (i.e., $17.4 \mathrm{Mpc}$ ), together with $v_{\mathrm{V}}=980 \mathrm{~km} \mathrm{~s}^{-1}$ (Teerikorpi et al. 1992) and $t_{0}=13.7 \mathrm{Gyr}$ (Spergel et al. 2003) as input parameters for the model and computing $M_{\mathrm{V}}$ as a result. In this way, we obtain a value of $M_{\mathrm{V}}=7 \times 10^{14} M_{\odot}$. Notice that the effective mass of the point mass model does not have to coincide with the virial mass of the cluster, as the latter one represents only the virialized part of the cluster, while the former gives us an idea of the dynamical mass of the whole Virgo region.

\section{Discussion}

As mentioned in Sect. 1 and clearly seen in Fig. 2, there is a non-negligible number of H I-deficient galaxies distant from the cluster core, in particular at distances of 10 and $28 \mathrm{Mpc}$ from the Local Group. We now examine different explanations for the presence of these $\mathrm{HI}$-deficient galaxies outside the core of Virgo:

1. very inaccurate distances (perhaps from the inadequacy of the Tully Fisher relation for galaxies undergoing ram pressure or tidal stripping) for galaxies that are in fact within the Virgo cluster (in the core or more probably in a rebound orbit) and which would have lost some of their gas by ram pressure stripping from the intracluster gas;

2. tidal interactions from members of a same group;

3. tidal interactions from neighbors;

4. recent minor mergers heating the gas;

5. overestimated H I-deficiency in early-type spirals.

In Table 7 are listed all galaxies from the compilation of Solanes et al. (2002) which satisfy at least one of the following criteria:

$D<13 \mathrm{Mpc}$,

$21<D<50 \mathrm{Mpc}$,

$\theta / \theta_{100}>1$

where $\theta$ is the angular distance to $\mathrm{M} 87$ and $\theta_{100}=r_{100} / D_{\mathrm{V}}=$ 5.6 is the angular virial radius of the Virgo cluster. Columns (1) and (2) give the galaxy names, the coordinates in Cols. (3) and (4), the galaxy type, total blue extinction corrected magnitude and optical diameter (all three from LEDA) are respectively in Cols. (5)-(7), the projected distance to M 87 in units of the virial radius $\left(r_{100}\right)$ of the cluster in Col. (8), the velocity relative to the Local Group taken from the Arecibo General 
Table 7. H I-deficient galaxies possibly outside the Virgo cluster.

\begin{tabular}{|c|c|c|c|c|c|c|c|c|c|c|c|c|}
\hline \multicolumn{2}{|c|}{ Galaxy } & RA & Dec & \multirow[t]{2}{*}{$T$} & \multirow[t]{2}{*}{$B_{T}^{c}$} & \multirow[t]{2}{*}{$D_{25}$} & \multirow[t]{2}{*}{$\theta$} & \multirow{2}{*}{$\begin{array}{c}v \\
\left(\mathrm{~km} \mathrm{~s}^{-1}\right)\end{array}$} & \multirow{2}{*}{$\begin{array}{c}D \\
(\mathrm{Mpc})\end{array}$} & \multirow[t]{2}{*}{$N$} & \multirow[t]{2}{*}{$P_{1}$} & \multirow[t]{2}{*}{$P_{2 .}$} \\
\hline VCC & NGC & \multicolumn{2}{|c|}{$(\mathrm{J} 2000)$} & & & & & & & & & \\
\hline- & 4064 & $12^{\mathrm{h}} 04^{\mathrm{m}} 11^{\mathrm{s}} .8$ & $18^{\circ} 26^{\prime} 33^{\prime \prime}$ & 1 & 11.73 & $4{ }^{\prime} 0$ & 8.8 & 837 & 9.8 & 942 & 0.00 & 0.81 \\
\hline 522 & 4305 & $12^{\mathrm{h}} 22^{\mathrm{m}} 03^{\mathrm{s}} .5$ & $12^{\circ} 44^{\prime} 27^{\prime \prime}$ & 1 & 12.87 & $2^{\prime} .0$ & 2.2 & 1814 & 40.7 & 200 & 0.00 & 0.00 \\
\hline 524 & 4307 & $12^{\mathrm{h}} 22^{\mathrm{m}} 06^{\mathrm{s}} .3$ & $09^{\circ} 02^{\prime} 27^{\prime \prime}$ & 3 & 11.84 & 3.5 & 4.0 & 913 & 27.4 & 1846 & 0.41 & 0.48 \\
\hline 559 & 4312 & $12^{\mathrm{h}} 22^{\mathrm{m}} 32^{\mathrm{s}} .0$ & $15^{\circ} 32^{\prime} 20^{\prime \prime}$ & 2 & 11.76 & $4 ! 7$ & 3.7 & 47 & 10.8 & 754 & 0.95 & 1.00 \\
\hline 713 & 4356 & $12^{\mathrm{h}} 24^{\mathrm{m}} 15^{\mathrm{s}} .9$ & $08^{\circ} 32^{\prime} 10^{\prime \prime}$ & 6 & 13.02 & $2: 6$ & $4^{\circ} .2$ & 998 & 29.4 & 1377 & 0.19 & 0.23 \\
\hline 979 & 4424 & $12 \mathrm{~h} 27^{\mathrm{m}} 13 \mathrm{~s} .3$ & $09^{\circ} 25^{\prime} 13^{\prime \prime}$ & 1 & 11.99 & $3: 4$ & 3.1 & 314 & 4.1 & 106 & 0.00 & 0.00 \\
\hline 1043 & 4438 & $12^{\mathrm{h}} 27^{\mathrm{m}} 46^{\varsigma} .3$ & $13^{\circ} 00^{\prime} 30^{\prime \prime}$ & 1 & 10.55 & $8: 7$ & 1.0 & -45 & 10.4 & 755 & 1.00 & 1.00 \\
\hline 1330 & 4492 & $12^{\mathrm{h}} 30^{\mathrm{m}} 58^{\mathrm{s}} .9$ & $08^{\circ} 04^{\prime} 41^{\prime \prime}$ & 1 & 13.04 & 1.9 & 4.3 & 1638 & 28.1 & 1261 & 0.08 & 0.11 \\
\hline 1569 & - & $12^{\mathrm{h}} 34^{\mathrm{m}} 31^{\mathrm{s}} .4$ & $13^{\circ} 30^{\prime} 23^{\prime \prime}$ & 5 & 14.62 & 0.8 & 1.4 & 687 & 23.3 & 8967 & 0.72 & 0.94 \\
\hline 1690 & $4569^{a}$ & $12^{\mathrm{h}} 36^{\mathrm{m}} 50^{\mathrm{s}} .5$ & $13^{\circ} 09^{\prime} 54^{\prime \prime}$ & 2 & 9.63 & $10^{\prime} .4$ & 1.7 & -328 & 9.4 & 143 & 1.00 & 1.00 \\
\hline 1730 & 4580 & $12^{\mathrm{h}} 37^{\mathrm{m}} 49^{\mathrm{s}} .5$ & $05^{\circ} 22^{\prime} 09^{\prime \prime}$ & 2 & 12.39 & $2^{\prime} \cdot 0$ & 7.2 & 893 & 19.3 & 8724 & 0.00 & 0.84 \\
\hline 1760 & 4586 & $12^{\mathrm{h}} 38^{\mathrm{m}} 28^{\mathrm{s}} .1$ & $04^{\circ} 19^{\prime} 09^{\prime \prime}$ & 1 & 12.06 & $3: 9$ & 8.3 & 639 & 14.4 & 4657 & 0.00 & 0.98 \\
\hline 1859 & 4606 & $12^{\mathrm{h}} 40^{\mathrm{m}} 57^{\mathrm{s}} .7$ & $11^{\circ} 54^{\prime} 46^{\prime \prime}$ & 1 & 12.28 & $2: 9$ & 2.5 & 1528 & 12.7 & 7648 & 0.95 & 1.00 \\
\hline
\end{tabular}

${ }^{a}$ NGC 4569 is also M 90.

Catalog (a private dataset maintained by Giovanelli and Haynes at the University of Cornell), in Cols. (9) and (10) gives the adopted distance (Solanes et al. 2002). The last 3 columns of Table 7 are explained in Sect. 4.1.

\subsection{Are the outlying $\mathrm{HI}$-deficient galaxies truly too far from the Virgo cluster to have had their gas ram pressure stripped?}

If one wishes to explain the outlying $\mathrm{HI}$-deficient galaxies as galaxies that have lost their interstellar gas by its stripping by ram pressure from the hot intracluster gas, then these galaxies must have passed through the core of the Virgo cluster where the intracluster gas is dense and the galaxy velocities large so that the ram pressure is largest.

Mamon et al. (2004) found that objects that have passed through the core of a structure in the past cannot be at distances greater than 1-2.5 virial radii from the cluster today. With their estimate of the distance $(16.8 \mathrm{Mpc})$ and the virial radius $(1.65 \mathrm{Mpc})$ of Virgo, this means that galaxies that have passed through the core of Virgo cannot lie at a distance from the Local Group greater than 18.5 or $20.9 \mathrm{Mpc}$ (for $r_{\mathrm{reb}} / r_{100}=1$ or 2.5, respectively) nor can they lie closer than 15.1 or $12.7 \mathrm{Mpc}$ from the Local Group.

For the $3 \mathrm{HI}$-deficient spirals at $28 \mathrm{Mpc}$ to have passed through the core of Virgo, one would require that their distances be each overestimated by $52 \%$ (34\%), which corresponds to $2.3 \sigma(1.6 \sigma)$ events for their distance moduli, for $r_{\text {reb }} / r_{100}=1(2.5)$, respectively. Such errors are possible, as shown in Sect. 3.5. Note that none of the distances of the 13 galaxies listed in Table 7 are based upon Cepheid measurements, which are much more precise than Tully-Fisher estimates. On the other hand, given the presence in our sample of some spirals with grossly incorrect distances (see Sect. 3.5), one may wonder if the distances to the 3 spirals at $28 \mathrm{Mpc}$ could also be grossly incorrect: they could lie at the cluster distance of $16.8 \mathrm{Mpc}$, thus leading in this case to distances overestimated by $66 \%$, which for $20 \%$ rms relative errors correspond to three $2.8 \sigma$ events for their distance moduli, which appears unlikely, unless the Tully-Fisher error distribution has non-Gaussian wings.

Similarly, the three foreground HI-deficient spirals at $\sim 10 \mathrm{Mpc}$ could have bounced out of the core of the Virgo cluster, as they lie on the outer edge of the envelope of the particles after inclusion of $20 \%$ rms distance errors in Fig. 3. Indeed, were they bouncing out of the Virgo cluster in the foreground at 15.1 (12.7) Mpc, one would then have three cases of $34 \%(21 \%)$ errors, each corresponding to $-2.3 \sigma(-1.3 \sigma)$ events for their distance moduli, for $r_{\mathrm{reb}} / r_{100}=1$ (2.5), respectively. Alternatively, they could lie in the cluster proper, at $16.8 \mathrm{Mpc}$, which would correspond to three $-2.8 \sigma$ events for their distance moduli, which again appears unlikely, unless the Tully-Fisher error distribution has non-Gaussian wings.

The offset of a galaxy relative to the Tolman-Bondi locus in Fig. 2 can be caused by a large distance error or a large peculiar velocity relative to the general flow at that distance, or a combination of both. To see which effect is more important, one can consider the $N$ simulated particles with distance (with errors folded in) and velocity respectively within $3 \mathrm{Mpc}$ and $200 \mathrm{~km} \mathrm{~s}^{-1}$ of each galaxy in Table 7, as well as angular distance to the halo center within 1.5 of that of the galaxy relative to M 87, and ask what fractions $P_{1}$ and $P_{2.5}$ of these particles were located (before the distance errors were folded in) within 1 or 2.5 times the virial radius of the halo, respectively.

These fractions must be taken with caution, because the groups falling in or moving out of the Virgo cluster are at different distances and with different relative masses between the simulations and the observations. For example, a true group of galaxies in the background of the Virgo cluster will end up with too high values of $P_{1}$ and $P_{2.5}$ if there is no group at the same 
distance in the simulations, while a Virgo cluster galaxy with a measured distance of $10 \mathrm{Mpc}$ or so beyond the cluster will have too low values of $P_{1}$ and $P_{2.5}$ if the simulations include a group at a distance close to this measured distance. Nevertheless, $P_{1}$ and $P_{2.5}$ should provide interesting first order constraints.

Of course, this method of locating galaxies, implies that galaxies NGC 4064, NGC 4580 and NGC 4586, at angular distances from M 87 beyond $\theta_{100}+1.5=7.1$ have $P_{1}=0$. Interestingly, all three galaxies are likely to be within $2.5 r_{100}$ (i.e. $P_{2}$ is close to unity).

Among the 5 foreground galaxies with $\theta<5: 6$, NGC 4312, NGC 4438, M90 and NGC 4606 are all likely to be in the Virgo cluster and all very likely to be close enough that they may have passed through its core. Only NGC 4424, apparently at distance $4.1 \mathrm{Mpc}$, is too close to actually be located within 2.5 virial radii from the Virgo cluster.

Among the 5 background galaxies with $\theta<5$. 6 , all but NGC 4305, lying at $40 \mathrm{Mpc}$, have a non negligible probability of having crossed the cluster. Only VCC 1569 measured at $23 \mathrm{Mpc}$ is highly likely to be located within the virial radius of the Virgo cluster. Interestingly, the 3 galaxies at measured distances around $28 \mathrm{Mpc}$ have very different values of $P_{1}$ and $P_{2.5}$. The closest one, NGC 4307, has one chance in two of being located near the cluster and $40 \%$ probability of being within the cluster itself. However, the farthest one, NGC 4356, has one chance in 5 of belonging to the Virgo cluster and less than one chance in four of being within 2.5 virial radii. The third one, NGC 4492, has only one chance in 9 of being within 2.5 virial radii of the Virgo cluster. This low probability is not surprising, given that it is located close to the infall/expansion zone of Fig. 2.

\subsection{Are the outlying $\mathrm{HI}$-deficient galaxies located within small groups?}

The alternative to ram pressure stripping of gas is removal of gas by tidal effects. One can envision various scenarios: 1) tidal stripping of gas beyond the optical radius; 2) tidal compression of gas clouds leading to gas transformation into stars; 3) tidal stripping of the gas reservoir infalling into the disk. Such tides can occur from interacting galaxies or from the cluster or group itself. We begin by asking whether the H I-deficient galaxies apparently outside of Virgo lie in groups.

Figure 4 shows the environment of the H I-deficient galaxies in three equal logarithm distance bins, $\pm 1.28 \times \sigma \ln D$ wide, centered around $10 \mathrm{Mpc}$ (the apparent distance to the foreground HI-deficient galaxies), $16.8 \mathrm{Mpc}$ (the distance to the Virgo cluster) and $28 \mathrm{Mpc}$ (the apparent distance to the background HI-deficient galaxies), as well as in three radial velocity bins. The log distance (velocity) bins are chosen wide enough that if the parent distribution function of the log distance (velocity) errors were Gaussian, one would have a probability $2 \operatorname{erf}(1.28)-1=0.86$ of having a galaxy with a true distance (velocity) within the interval appearing to lie at the center of the interval.

Among the 5 foreground HI-deficient spirals, 4 (NGC 4312, NGC 4438, M 90, which, as noted in Sect. 3.5, is far off the Tolman-Bondi relation, and NGC 4606), appear superposed (within dotted circle in upper-left plot) with the galaxies at the distance of the main structure of the Virgo cluster, which suggests that their distances may be seriously underestimated, unless there happens to be a group of galaxies precisely along the line of sight to the center of Virgo lying at $10 \mathrm{Mpc}$.

One may be tempted to identify the $3 \mathrm{HI}$-deficient spirals close to M 87 (NGC 4438, M 90, and NGC 4606) with a group of ellipticals seen in the upper-left plot of Fig. 4. The velocity dispersion of this group would explain why these 3 galaxies are so far off the Tolman-Bondi solution given in Fig. 2. However, none of the ellipticals in this region have accurate $(<38 \%)$ relative distance estimates (cf. Table 2) and some or all may actually belong to the Virgo cluster.

Interestingly, 3 of the $4 \mathrm{HI}$-deficient spirals at $28 \mathrm{Mpc}$ (NGC 4307, NGC 4356 and NGC 4492) lie in a dense region, extending to the SSW of the position of M 87 (lower-left plot), which is also seen in the middle distance bin (middle-left plot) corresponding to the distance to M 87, but with 3 times fewer galaxies. Although the eastern edge of the region, and particularly the HI-deficient NGC 4492, coincide with the position of M 49 (NGC 4472), it appears distinct from M 49, which roughly lies at the middle of a group of galaxies (middle-left plot) that does not seem to be associated with the dense region at $28 \mathrm{Mpc}$ (lower-left plot).

We now compare the Tully-Fisher distance estimator with the redshift distance estimator $D_{v}=v / H_{0}$. If a structure outside of the Virgo cluster lies at a distance $D$ within a region with $1 \mathrm{D}$ velocity dispersion $\sigma_{v}$, the error on the distance estimator $D_{v}=v / H_{0}$ will be set by the dispersion of the peculiar velocities:

$\delta D_{v}=\sigma_{v} / H_{0}$.

The redshift distance estimator $D_{v}$ will be more accurate than the direct distance estimator when

$H_{0} D>\frac{\sigma_{v}}{\delta D / D}$

where $\delta D / D$ is the relative error of the direct distance estimator. For Tully-Fisher distance measurements, $\delta D / D \simeq 20 \%$, and a field velocity dispersion of $40-85 \mathrm{~km} \mathrm{~s}^{-1}$ (as observed by Ekholm et al. 2001 and Karachentsev et al. 2003, as well as predicted by Klypin et al. 2003), Eq. (12) yields a maximum distance for believing individual direct distances of 3 to $7 h_{2 / 3}^{-1} \mathrm{Mpc}$, so that individual galaxy distances of $28 \mathrm{Mpc}$ are much less reliable.

This change in optimal distance estimators is illustrated in Fig. 1, where only one of the background H I-deficient galaxies has a radial velocity much larger than the mean of the Virgo cluster. The plots on the right of Fig. 4 show that the structure to the south-southwest (SSW) of the Virgo cluster lies close to Virgo in velocity space (i.e. the concentration is in the right middle plot). In redshift space, the 3 background $\mathrm{H}$ I-deficient galaxies to the SSW of M 87 remain in a similar concentration of galaxies (middle right plot of Fig. 4) as in distance space (lower left plot), but this concentration is at similar radial velocities as the Virgo cluster. However, only one (NGC 4307) has a 

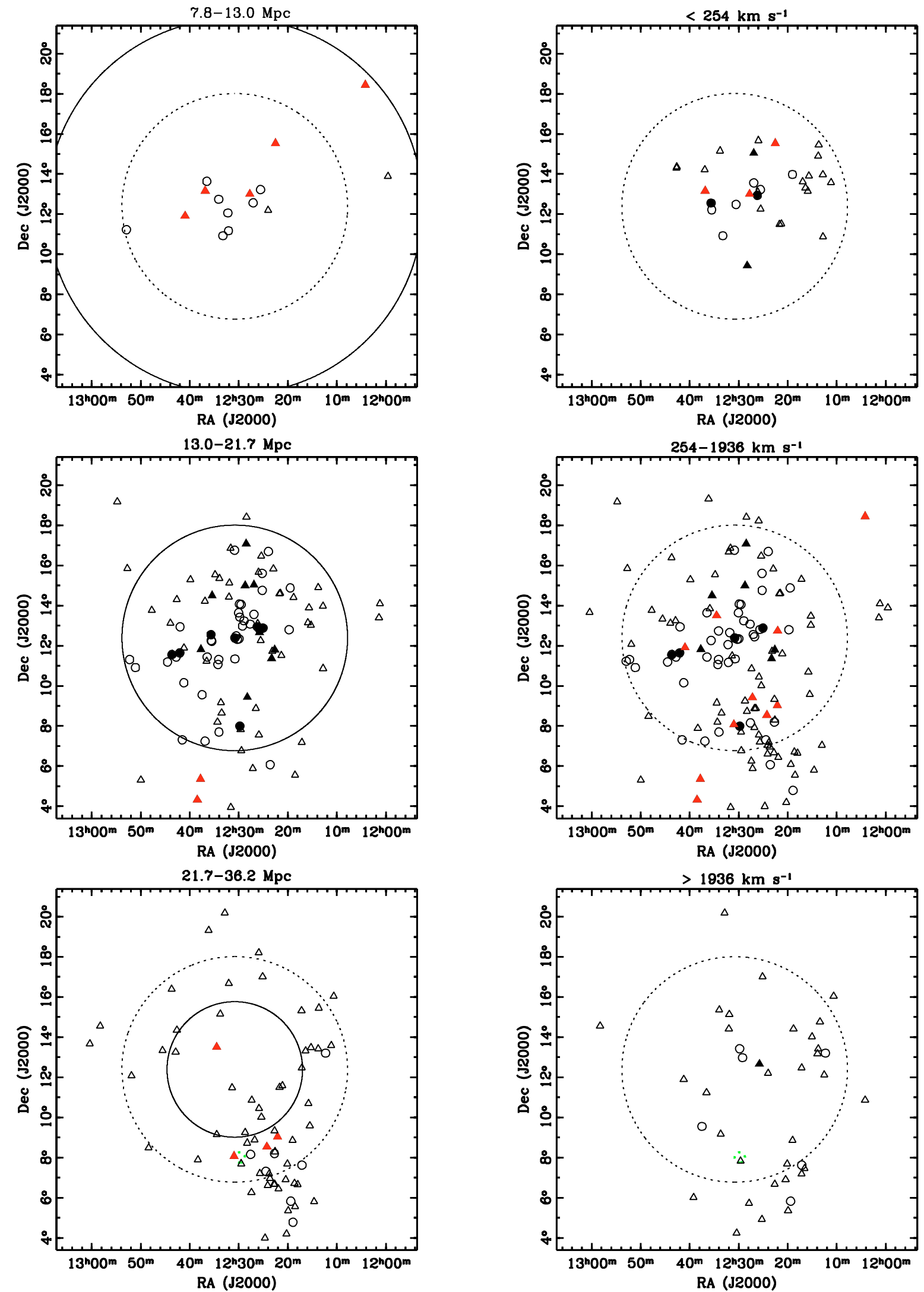

Fig. 4. Positions of galaxies within $9^{\circ}$ of $\mathrm{M} 87$ in 3 distance (left) and velocity (right) bins. Ellipticals and spirals are shown as circles and triangles, respectively. Messier ellipticals are shown as filled circles, while M 49 (NGC 4472) is also highlighted as a thick dotted circle (bottom plots, in green in the electronic version of the journal). H I-deficient $(>3 \sigma)$ spirals are shown as filled triangles (with the 13 apparently in the outskirts of Virgo (Table 7) in red in the electronic version of the Journal). The large solid circles show the virial radius $r_{100}$ at the different distance bins, while the large dotted circles indicates $r_{100}$ at the distance to M 87. 
Table 8. Companions to H I-deficient galaxies in the outskirts of Virgo.

\begin{tabular}{|c|c|c|c|c|c|c|c|c|}
\hline \multicolumn{2}{|c|}{ Galaxy } & \multicolumn{2}{|c|}{ Companion } & \multirow{2}{*}{$\begin{array}{c}\Delta v \\
\left(\mathrm{~km} \mathrm{~s}^{-1}\right)\end{array}$} & \multirow[t]{2}{*}{$\Delta m_{T}^{c}$} & \multirow[t]{2}{*}{$d / r_{\mathrm{opt}}$} & \multirow[t]{2}{*}{$\mathrm{M}$} & \multirow[t]{2}{*}{ K } \\
\hline VCC & NGC & VCC & NGC & & & & & \\
\hline 522 & 4305 & 523 & 4306 & -380 & $0.9^{a}$ & 2.8 & $\mathrm{R}$ ? & - \\
\hline 1043 & $4438^{b}$ & 1030 & 4435 & -214 & 1.0 & 1.0 & I & I \\
\hline 1690 & $4569^{c}$ & $1686^{d}$ & - & 1354 & 4.2 & 1.1 & $\mathrm{R}$ & $\mathrm{R}$ \\
\hline 1859 & 4606 & 1868 & 4607 & 593 & 0.6 & 2.7 & $\mathrm{I} ?$ & - \\
\hline
\end{tabular}

Notes: ${ }^{a} B$-band; ${ }^{b}$ also Arp $120 ;{ }^{c}$ also M90 and Arp 76; ${ }^{d}$ also IC 3583 .

reasonably high chance $\left(P_{1}\right.$ in Table 7$)$ of being located in or near the Virgo cluster, while one (NGC 4356) has less than one chance in 4 of being in or near the cluster (from the analysis of Sect. 4.1), and one (NGC 4492) has a radial velocity indicative of a background object expanding away from the Virgo cluster (this galaxy shows up as the empty triangle in Fig. 2 very close to the Tolman-Bondi locus), and has only roughly one chance in 9 of being even close to the cluster. But the analysis of $P_{1}$ and $P_{2.5}$ of Sect. 4.1 should be superior to the analysis of Fig. 4 , because it takes into account the correlation of distance and velocity. In summary, the 3 background H I-deficient galaxies to the SSW of M 87 may be suffering from tidal effects of a background group of galaxies.

\subsection{Close companions and mergers}

According to a search in SIMBAD, we find that (at least) 4 of the $13 \mathrm{HI}$-deficient galaxies in Table 7 have companions whose mass and proximity may generate tides that could remove some of the neutral hydrogen, either by stripping the gas beyond the optical radius (without stripping the stars, thus leaving an H I-deficiency) or by compressing the diffuse neutral hydrogen, converting atomic gas into molecular gas, then into stars. Indeed, galaxies in interacting pairs have a high molecular to atomic gas ratio (Combes et al. 1994). Of course, the presence of a companion in projection, even with a similar velocity, does not guarantee that this companion is in physical interaction with the galaxy in question, as projection effects are important in clusters.

Table 8 shows the properties of the companions to these 4 galaxies. Column 5 is the difference in velocity relative to the Local Group; Col. 6 is the difference in extinction-corrected total $I$-band magnitude; Col. 7 is the angular distance between the two components, in units of the optical radius $\left(D_{25} / 2\right)$ of the major member; Cols. 8 and 9 are indicators for morphological and kinematical (Rubin et al. 1999) disturbances $(\mathrm{R} \rightarrow$ regular, I $\rightarrow$ irregular) for the major component. Table 8 indicates that NGC 4438 has a very close major companion, which is likely to be responsible for its irregular morphology and internal kinematics. NGC 4305 and NGC 4606 have moderately close major companions. M 90 has a close very minor $(\approx 1 / 50$ in mass) companion.

Moreover, NGC 4307 and NGC 4356 are close in 4D space: their projected separation (at the distance of M 87) is $215 \mathrm{kpc}$ and their velocity difference is $85 \mathrm{~km} \mathrm{~s}^{-1}$, so they may constitute an interacting pair. Similarly, NGC 4580 and NGC 4586 are at a projected separation of $311 \mathrm{kpc}$ with a velocity difference of $254 \mathrm{~km} \mathrm{~s}^{-1}$, so they too may constitute an interacting pair.

Finally, two other galaxies in Table 7 have unusually low inner rotation (Rubin et al. 1999): NGC 4064 and NGC 4424. Kenney et al. (1996) argue that NGC 4424 has undergone a merger with a galaxy 2 to 10 times less massive and presumably the same can be said for NGC 4064.

We can obtain some clues about the origin of the HI deficiency of the outlying galaxies by studying their star formation rates. We adopt the, obviously simplified, point of view that tidal interactions are believed to enhance star formation (e.g. Liu \& Kennicutt 1995), while ram pressure stripping should reduce star formation. We therefore verify the tidal interaction hypothesis by checking for enhanced star formation rates. Because tidal interactions operate on scales of order the orbital times of interacting pairs, typically $250 \mathrm{Myr}$ or more, we do not use the $\mathrm{H} \alpha$ star formation rate indicator, as it is only sensitive to very recent star formation, but focus instead on the broadband color $B-H$. We have taken $B$ and $H$ magnitudes from the GOLDMine database (Gavazzi et al. 2003).

Figure 5 shows the $B-H$ colors as a function of $H$-band luminosity for the 12 NGC galaxies of Table 7 (we have omitted VCC 1569, which is too underluminous to be in GOLDMine) and for other Virgo galaxies. The colors and luminosities in this figure were corrected for absorption using internal and galactic (Schlegel et al. 1998) absorption obtained from LEDA, assuming selective extinction coefficients from Cardelli et al. (1989). Figure 5 clearly displays a general color-luminosity relation, i.e. the trend that more luminous galaxies are redder. Comparing galaxies of similar mophological types, one checks that among the four candidates for possessing tidal companions, NGC 4305, NGC 4438, NGC 4569 (M90), and NGC 4606 (see Table 8), the first three are blue for their luminosities, assuming their Tully-Fisher distances, but given that the latter three might well lie in Virgo (see Col. $P_{1}$ in Table 7), we also check that at the distance to the Virgo cluster these 3 galaxies are very blue (NGC 4438), extremely blue (M90) and just blue (NGC 4606). The blue colors for these 4 galaxies, given their luminosity and morphological type, confirms the tidal hypothesis for all four objects.

Interestingly, the possibly interacting galaxies NGC 4307 and NGC 4356 have blue colors confirming their interaction if they are indeed in the background of Virgo as their TullyFisher distances suggest, but have red colors as expected for ram pressure stripped galaxies if they lie in the Virgo cluster. The values of $P_{1}$ in Table 7 cannot distinguish between these two possibilities. The situation is even more uncertain for the possibly interacting galaxies NGC 4580 and NGC 4586, whose Tully-Fisher distances place them slightly behind and in front of the Virgo cluster, respectively. If they both lie at the distance of the Virgo cluster, the first one would have a normal color, while NGC 4586 would be blue as expected if it is indeed interacting with NGC 4580.

The situation is also confusing for the two galaxies with possible recent minor merging: NGC 4064 appears normal or red if it lies in the foreground as suggested by its Tully-Fisher 


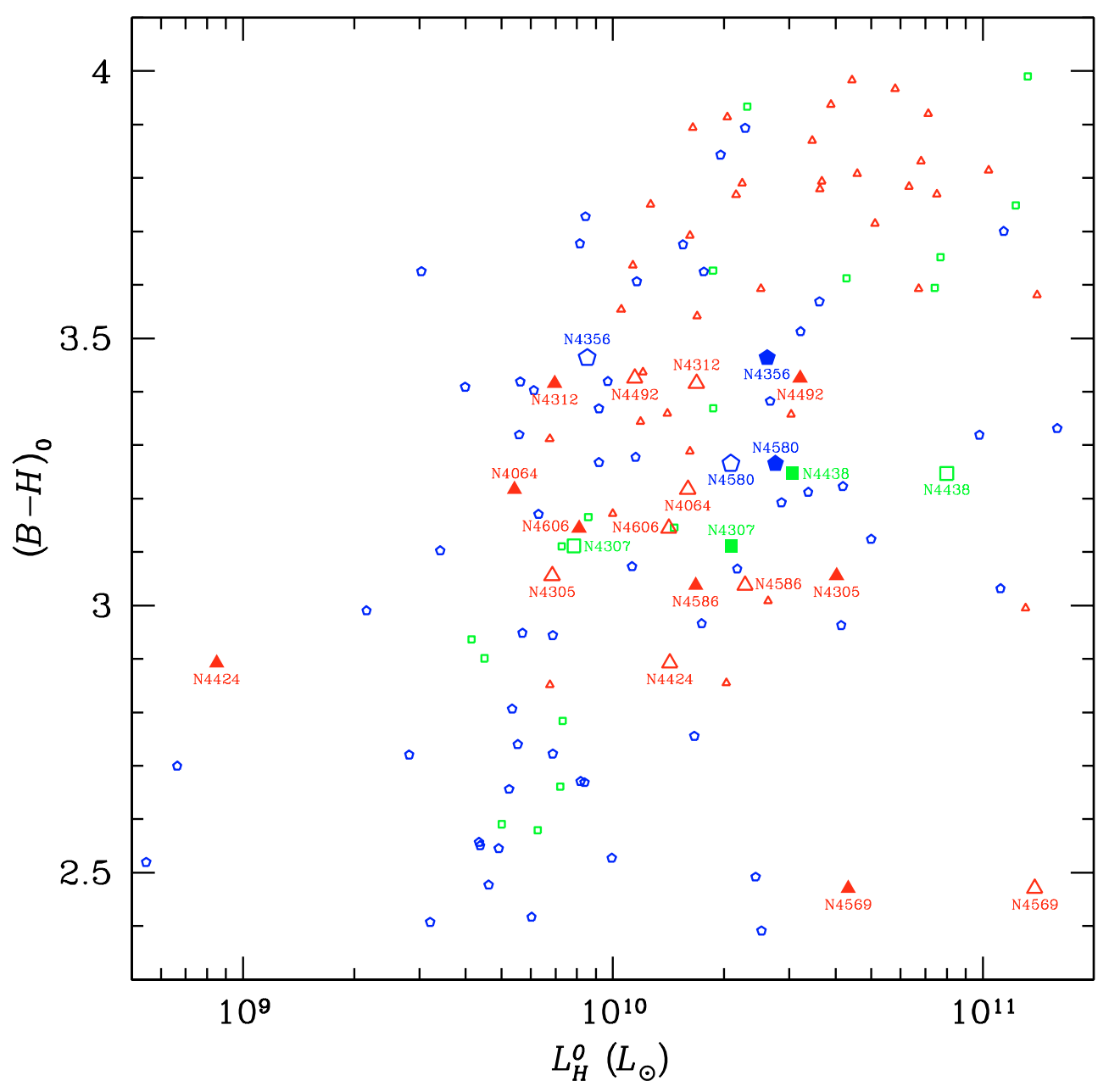

Fig. 5. Color-luminosity diagram for 12 of the $13 \mathrm{H}$ I-deficient galaxies of Table 7 (VCC 1569 was excluded because it is not bright enough to be included in GOLDMine), possibly outside the Virgo cluster (large filled symbols) or placed at the distance of the Virgo cluster (large open symbols). Other Virgo galaxies are shown as small open symbols. Triangles, squares and pentagons (respectively in red, green, and blue in the electronic version of the journal) refer to galaxies of morphological type 1-2,3-4 and 5-6, respectively. The data is taken from the GOLDMine (Gavazzi et al. 2003) database, and dereddened using the internal and Galactic extinctions from LEDA with the selective extinctions of Cardelli et al. (1989).

distance, but blue if it lies at the distance of the Virgo cluster, and NGC 4424 appears normal or red if it lies as close as its Tully-Fisher distance suggests but quite blue if it lies at the distance of Virgo. One would expect the minor merger to generate a blue nucleus if the merging took place recently enough, but this may have little effect on the overall color of the galaxy.

Moreover, one may contest that tides will remove stars as well as gas from the disk of a spiral galaxy. However, the estimates of H I-deficiency presented here are based upon singledish (Arecibo) radio observations, which often do not have sufficient angular resolution to map the gas (the half-power beam of Arecibo subtends 3.3, close to the typical optical angular diameters of the HI-deficient galaxies - see Table 7). One can therefore easily imagine that fairly weak tidal effects will deplete the neutral hydrogen gas beyond the optical radius without affecting the stars in the spiral disk, thus leading to an $\mathrm{H}$ I-deficiency when normalized relative to the size of the optical disk.

Two galaxies among our H I-deficient Virgo outliers listed in Table 7 (NGC 4438 and M90) have been studied by
Cayatte et al. (1994), who computed H I-deficiencies of Virgo spirals, based upon high resolution $21 \mathrm{~cm}$ VLA observations. Both galaxies present very small $21 \mathrm{~cm}$ to optical diameter ratios, indicating that the gas deficiency sets in at small radii and cannot be caused by tidal effects. Additional high-resolution $21 \mathrm{~cm}$ observations at the VLA of the other galaxies in Table 7 will obviously allow confirmation of this result.

\subsection{Are the outlying $\mathrm{HI}$-deficient galaxies truly gas-deficient?}

The H I-deficiency estimator has been defined by several authors (e.g. Chamaraux et al. 1980; Haynes \& Giovanelli 1984; Solanes et al. 1996) as the hydrogen mass relative to the mean for galaxies of the same morphological type and optical diameter:

$\mathrm{DEF}_{1}=\left\langle\log M_{\mathrm{HI}}\left(D_{\mathrm{opt}}, T\right)\right\rangle-\log M_{\mathrm{HI}}$,

where $M_{\mathrm{HI}}$ is the H I mass of the galaxy in solar units. Solanes et al. (2002) applied a different H I-deficiency estimator based 


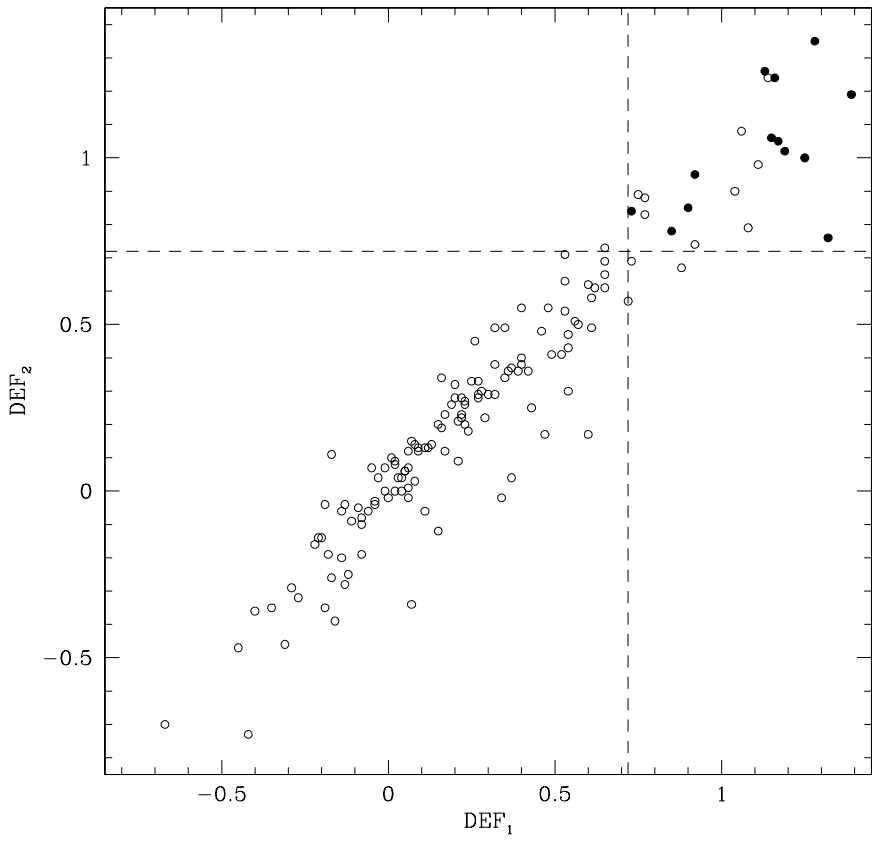

Fig. 6. Comparison of H I-deficiency estimators. Galaxies possibly located outside the Virgo cluster are shown as filled circles. The vertical and horizontal dashed lines shows the $3 \sigma$ cutoff for $\mathrm{DEF}_{1}$ and $\mathrm{DEF}_{2}$, respectively.

on the hybrid mean surface brightness expected for each galaxy type independently of its diameter, which has the advantage of being independent of the distance to the galaxies:

$\mathrm{DEF}_{2}=\left\langle\log \bar{\Sigma}_{\mathrm{HI}}(T)\right\rangle-\log \bar{\Sigma}_{\mathrm{HI}}$,

where $\bar{\Sigma}_{\mathrm{HI}}$ is the hybrid mean surface brightness, defined as

$\bar{\Sigma}_{\mathrm{HI}}=\frac{F_{\mathrm{HI}}}{\theta_{\mathrm{opt}}^{2}}$,

where $F_{\mathrm{HI}}$ and $\theta_{\mathrm{opt}}$ are the $\mathrm{HI}$ flux and optical angular diameter, respectively.

Could the seemingly HI-deficient galaxies in the foreground and background of Virgo have normal gas content according to Eq. (13) but appear deficient with Eq. (14)? Solanes et al. checked the statistical agreement of these two $\mathrm{H}$ I-deficiency estimators by fitting a linear model between the two parameters and found a regression line with slope and intercept close to the unit and zero respectively, with a scatter of 0.126 . Figure 6 shows this correlation, where those galaxies possible located outside the Virgo cluster are shown as solid circles. The plot clearly indicates that the outliers are also deficient with the $\mathrm{DEF}_{1}$ definition of H I-deficiency.

However, Table 7 clearly shows that 7 out of our 13 H I-deficient possible outliers appear to be Sa galaxies $(T=$ 1), some of which might be misclassified S0/a or even S0 galaxies, which are known to have little cold gas. The stronger H I-deficiency of early-type spirals in the Virgo cluster has been already noticed by Guiderdoni \& Rocca-Volmerange (1985). Some of these Sa galaxies may not be deficient relative to field S0/a or S0 galaxies. Indeed, the most recent study (Bettoni et al. 2003) of the H I content of galaxies as a function of morphological type indicates that the logarithm of the H I mass normalized to luminosity or to square optical diameter decreases increasingly faster for earlier galaxy types, therefore its slope for Sa's is considerably larger than for Sc's, and hence S0/a galaxies misclassified as Sa's will appear more $\mathrm{HI}$-deficient than later-type galaxies misclassified by one-half of a morphological type within the Hubble sequence.

Moreover, independent estimates of morphological types by experts led to $\sigma(T)=1.5$ (rms) for galaxies with optical diameters $>2^{\prime}$ (Naim et al. 1995). The galaxies listed in Table 7 have optical diameters ranging from $2^{\prime}$ to $10^{\prime}$ plus one (VCC 1569) with $D=0$ ' 8 . There are two galaxies (NGC 4305 and NGC 4492) with $T=1$ and $D \leq 2^{\prime}$, i.e. where the uncertainty on $T$ is probably as high as 1.5 .

For example, NGC 4424 and NGC 4492 are marginally $\mathrm{HI}$-deficient and are prime candidates for losing their status of gas-deficient if they are misclassified lenticulars (by $\Delta T=1$, the typical scatter given by LEDA).

We must thus resort to visualizing the possibly misclassified galaxies. Figure 7 displays the snapshots of the $7 \mathrm{HI}$-deficient outliers classified as $\mathrm{Sa}(T=1)$. We now discuss the morphologies of these 7 galaxies.

NGC 4064 This galaxy has a very large high surface brightness inner disk or flattened bulge with little trace of spiral arms. NGC 4064 may lie between S0 and S0/a.

NGC 4305 This galaxy is of type $\mathrm{Sa}$ or later. It has a nearby major companion.

NGC 4424 This galaxy has an important bar and the size of the bulge is difficult to estimate (Kenney et al. $1996 \mathrm{fit}$ the bulge-disk ratio to between 0.2 and 0.6 ). It shows little traces of spiral arms. Kenney et al. write "NGC 4424 is classified as an Sa rather than an S0 because the outer stellar disk is not structureless". It may well be an S0/a.

NGC 4438 This galaxy, with a large bulge, is tidally distorted by a companion just North of the frame. Gavazzi et al. (2000) fit a pure $r^{1 / 4}$ law to this galaxy, suggesting that its morphology is of an earlier type than Sa.

NGC 4492 This galaxy is viewed face-on, hence the disk is not very visible. The bulge is relatively large and there are signs of a weak spiral arm. NGC 4492 may lie between S0/a and $\mathrm{Sa}$, but Gavazzi et al. (2000) fit a bulge to disk ratio of $1 / 3$, suggesting a type of Sa or later.

NGC 4586 This galaxy shows spiral arms and a normal bulge for an Sa galaxy.

NGC 4606 This galaxy shows little traces of spiral arms and has an intermediate bulge. NGC 4606 appears between S0/a and Sa. However, Gavazzi et al. (2000) fit a bulge to disk ratio of $1 / 20$, suggesting a later type than $\mathrm{Sa}$.

Altogether, we suspect that 2 galaxies may be earlier than Sa: NGC 4064 and NGC 4424, both of which appear to have recently ingested smaller galaxies, and significant amounts of cold gas may have been heated up during the merger.

Given that between types S0/a and Sa, the compilation of Bettoni et al. (2003) (left plots of their Fig. 2) indicates that the normalized HI content varies as $\log M_{\mathrm{HI}}^{\text {norm }} \sim T / 4+$ cst, an overestimate of $1(0.5)$ in $T$ will lead to an underestimate in H I-deficiency of 0.25 (0.125). The H I-deficiency of NGC 4064 is sufficiently high that a correction of 0.25 will still keep it 

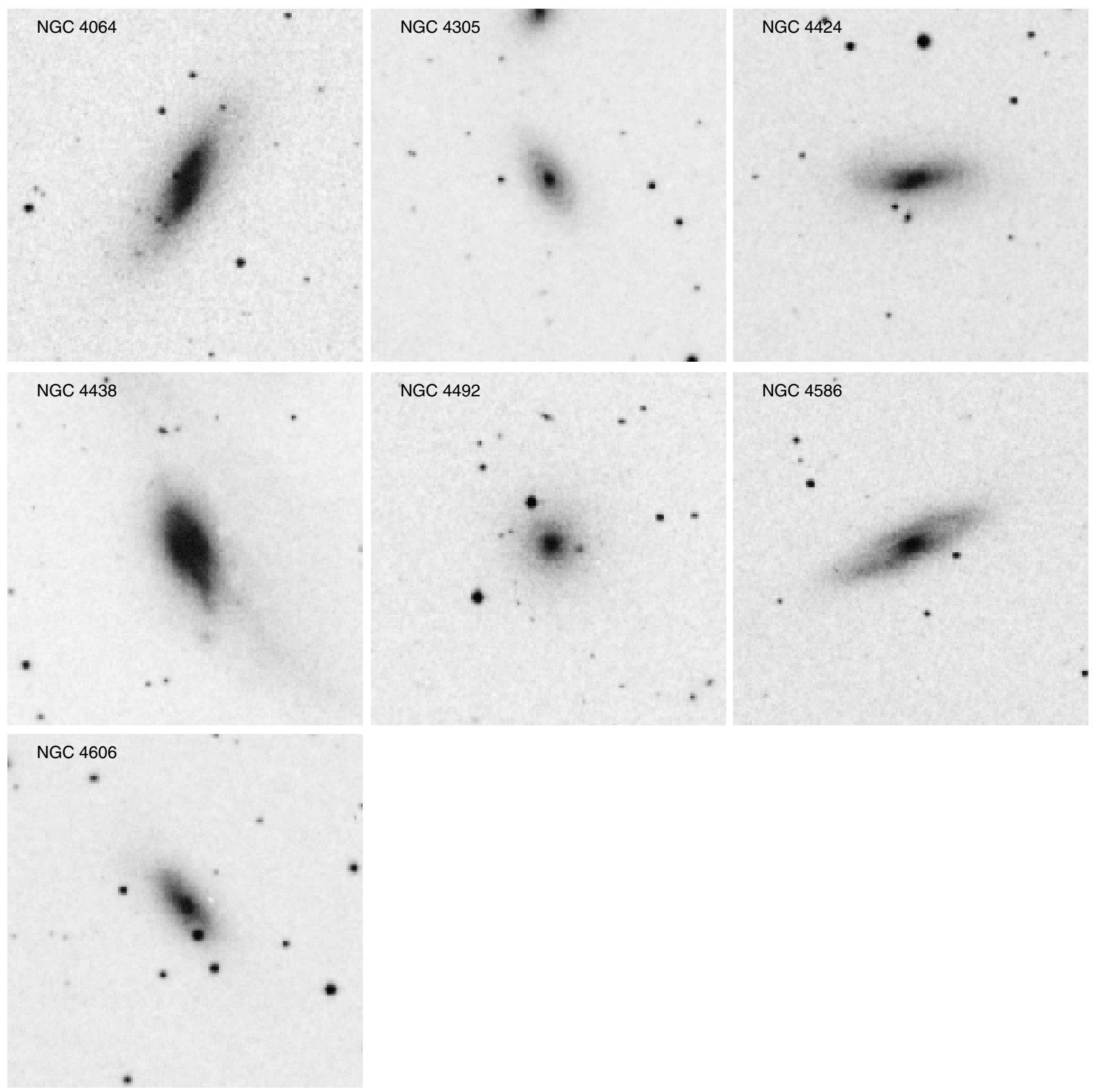

Fig. 7. POSS I snapshots of the $T=1 \mathrm{H}$ I-deficient galaxies of Table 7. The box size is $6^{\prime}$ and North points upwards.

among the $3 \sigma$ cases using both $\mathrm{DEF}_{1}$ and $\mathrm{DEF}_{2}$ criteria. On the other hand, if the morphological type of NGC 4424 is S0/a $(T=0)$, it would still be $\mathrm{H}$ I-deficient with criterion $\mathrm{DEF}_{1}$, but no longer among the $3 \sigma$ cases with $\mathrm{DEF}_{2}$.

Note, however, that if most H I-deficient outliers classified as Sa's were in fact S0/a's, we would find ourselves with the new problem of justifying the presence of S0/a's so far away from the cluster core and apparently not within groups (see Sect. 5.3). We also have to keep in mind that Koopmann \& Kenney (1998) argued that spirals in the Virgo cluster could have also been misidentified as early-type objects, an opposite trend to the above suggested.

\subsection{Case by case analysis}

We now focus on each of the 13 galaxies, one by one.

NGC 4064 The very low inner rotation (Rubin et al. 1999) suggests that it is a merger remnant (see NGC 4424 below), which may have been responsible for its H I-deficiency.

NGC 4305 This galaxy, at $40 \mathrm{Mpc}$ and with a large radial velocity, is almost certainly a background galaxy. It has a major tidal companion (Table 8) and a blue color for its luminosity, assuming it is indeed far behind Virgo, confirming the tidal origin of its gas deficiency. 
NGC 4307 The origin of the H I-deficiency in this galaxy is difficult to ascertain, as it has $41 \%$ (48\%) probability of lying within the sphere centered on the Virgo cluster with radius 1 (2.5) times the virial radius. Its proximity with NGC 4356 suggests that it may have interacted in the past with that galaxy, and if it is indeed in the background of Virgo, as suggested by its Tully-Fisher distance, it would be blue for its luminosity, confirming the interaction hypothesis with NGC 4356.

NGC 4312 This galaxy is very likely within the Virgo cluster.

NGC 4356 The origin of the HI-deficiency in this galaxy is difficult to ascertain, as it has $19 \%$ (23\%) probability of lying within the sphere centered on the Virgo cluster with radius 1 (2.5) times the virial radius. Its proximity with NGC 4307 suggests that it may have interacted in the past with that galaxy, as confirmed by its blue color for its luminosity, assuming it lies indeed in the background of Virgo.

NGC 4424 The very low inner rotation of this galaxy suggests a merger remnant (Rubin et al. 1999). It almost certainly lies in the foreground of the Virgo cluster. Moreover, as discussed in Sect. 4.4, NGC 4424 may be of type S0/a, in which case it would not be $3 \sigma$ deficient in neutral hydrogen.

NGC 4438 This seemingly foreground galaxy is very probably a member of the Virgo cluster. Kotanyi \& Ekers (1983) suggest that the HI-deficiency of this highly distorted galaxy is caused not by the tidal companion, which should increase the line-width, but by interaction with the intracluster medium of the Virgo cluster. Moreover, this galaxy has a very small radial extent of its HI gas, as attested by the VLA map of Cayatte et al. (1994). Interestingly, at the distance of Virgo, this galaxy would be very blue, which is easily explained by the presence of its tidal companion.

NGC 4492 This galaxy is probably in the background of the Virgo cluster, in which case it would be blue for its luminosity and morphological type. It may lie in a group that could include NGC 4307 and NGC 4356 and thus be suffering tidal encounters with these and/or other members of the group.

VCC 1569 This faint galaxy probably lies in or very close to the Virgo cluster.

M90 Among the 13 galaxies listed in Table 7, this very blue Sab LINER (e.g. Keel 1996) deviates the most significantly from both the Tolman-Bondi locus (Fig. 2) and from the color-luminosity diagram (Fig. 5). Its distance has been controversial. Stauffer et al. (1986) suggest that the TullyFisher estimate is an underestimate because the optical rotation curve is still rising to the limit of its measurement (see Rubin et al. 1999) and that similarly low distances derived by Cowley et al. (1982) using their line index/bulge luminosity relation may be erroneous because the weakness of the nuclear lines is in contrast with the high metallicity expected from the stellar population of the bulge.

M90 is extremely isolated in projected phase space. Among the galaxies with $D<13 \mathrm{Mpc}$, it appears associated with a group of ellipticals, none of which has accurate distance measurements, with sky coordinates associated with the central ellipticals with accurate distance measurements in the center of the Virgo cluster. Given its bright corrected total blue magnitude of 9.63 (Table 7), its absolute magnitude would correspond to $M_{B}=-20.24$ at $9.4 \mathrm{Mpc}$ or $M_{B}=-21.5$ at $16.8 \mathrm{Mpc}$. The latter absolute magnitude is very bright for a spiral galaxy. This can be quantified by comparing with the turnover luminosity, $L_{*}$, of the field galaxy luminosity function. Unfortunately, the surveys used to derive the field galaxy luminosity function do not employ the Johnson $B$ band used here. However, the 2MASS survey has measured a total $K$-band magnitude of $K=6.58$ for M 90. For a turnover absolute magnitude (Kochanek et al. 2001) $M_{K}^{*}=-23.39+5 \log h=-24.27$ for $h=2 / 3$ adopted here, the $K$-band luminosity of M 90 corresponds to $0.4 L_{*}$ if M 90 is at $9.4 \mathrm{Mpc}$ but $1.3 L_{*}$ if it lies at the distance of the Virgo cluster, which is a large luminosity for a spiral galaxy. Moreover, at the distance of Virgo, M90 would be extremely blue for its luminosity (Fig. 5).

Finally, the contours of continuum radio emission seen from the NVSS survey taken at the VLA are compressed in the East-Northeast direction, which is opposite to the direction towards the center of the Virgo cluster. This suggests that M 90 may be bouncing out of the cluster, which would also explain the galaxy's negative radial velocity.

NGC 4580 This galaxy probably lies near but not in the Virgo cluster, and may possibly be bouncing out of the cluster, therefore losing its neutral gas by ram pressure stripping. It may be interacting with NGC 4586, and is somewhat blue for its luminosity, especially if it lies behind Virgo.

NGC 4586 This galaxy probably lies near the Virgo cluster and may possibly be bouncing out of the cluster, therefore losing its neutral gas by ram pressure stripping. It may be interacting with NGC 4580, and is blue for its luminosity, especially if it lies within the Virgo cluster.

NGC 4606 This galaxy is a very likely member of the Virgo cluster. It is likely to be tidally perturbed by its companion NGC 4607 (Table 8), as attested by its blue color for its luminosity assuming it indeed lies in Virgo, although it shows only modest signs of such tides.

\subsection{Origin of the $\mathrm{HI}$-deficiency in the outlying galaxies}

The HI-deficient galaxies apparently lying well in front and behind of the Virgo cluster must have passed through the core of the cluster to have shed their gas by stripping by the ram pressure of the intracluster gas. As shown by Mamon et al. (2004), galaxies cannot bounce out beyond 1 to 2.5 virial radii, which according to their estimate of the virial radius of Virgo, amounts to 1.7 to $4.1 \mathrm{Mpc}$. Therefore, given a distance to the Virgo cluster of $16.8 \mathrm{Mpc}$, we suggest that only galaxies with distances between 12.7 and $20.9 \mathrm{Mpc}$ could have passed through Virgo.

One possibility, discussed in Sect. 4.1, is that the true 3D locations of the outlying $\mathrm{HI}$-deficient galaxies are in fact within the Virgo cluster's virial radius or at most within $4 \mathrm{Mpc}$ from its center, which is possible (although not likely) given the typical 20\% relative distance errors for the spiral galaxies in Virgo, 
expected from the Tully-Fisher relation, and from our comparison of the observed velocity distance relation with that derived from cosmological $N$-body simulations. Indeed, of the $13 \mathrm{HI}$-deficient spirals appearing in the outskirts of the Virgo cluster, 4 to 5 (depending on how to place the distance to M 90) are highly likely to be located in 3D within the virial radius of the Virgo cluster (Table 7) and one other galaxy (NGC 4307) has one chance in two of being in the cluster or near it. Three others are at an angular radius $>\theta_{100}$ from M 87, but appear to be within 2.5 virial radii of it in 3D. Finally, two galaxies (NGC 4356 and NGC 4492) in the direction of the cluster have low but non negligible probability of being in or near the cluster, while the last two are definitely foreground (NGC 4424) and background (NGC 4305) galaxies. Hence, between 4 and 11 of these 13 galaxies may have passed through the core of the Virgo cluster and seen their neutral gas ram pressure swept by the hot intracluster gas.

The presence of a few discrepant distance measurements suggests that the nearly Gaussian parent distribution function for measurement errors in the distance modulus (or relative distance) has extended non-Gaussian wings.

It is also possible that highly inaccurate (at the $30 \%$ level or more) Tully-Fisher distance measurements may arise from physical biases. If galaxies are H I-deficient because of strong interactions with the cluster (through ram pressure stripping or tides) or with neighboring galaxies (through collisional tides), their Tully-Fisher distances may be biased and/or inefficient. Indeed, our analysis of Sect. 4.3 indicates that, among the galaxies likely or possibly within the Virgo cluster, one (NGC 4438) shows clear tidal perturbations from a close major companion, while 2 others (M 90 and NGC 4606) are probably tidally perturbed by close companions, and the one possible Virgo cluster member (NGC 4307) may have interacted with a fairly close major companion in the past.

Moreover, there are general arguments, independent of these case-study tidal effects, that suggest that abnormal TullyFisher distances may be caused by HI-deficiency. For example, Stauffer et al. (1986) noted that H I-deficient spirals in the direction of the Virgo cluster tend to have smaller line-widths for their $H$-band luminosity, and, similarly, Rubin et al. (1991) found that the maximum rotation velocity of spiral galaxies within compact groups of galaxies tend to be smaller for their luminosity than for normal spirals. Such small line-widths or maximum rotation velocities imply that distances are underestimated. Using 2D (Fabry-Perot) spectroscopy, Mendes de Oliveira et al. (2003) have refined the analysis of Rubin et al. and find that the lowest luminosity galaxies in their sample $\left(M_{B}=-19.5 \pm 1\right)$ have lower maximum rotation velocities than field spirals of the same luminosity. Moreover, they find that compact group galaxies display a more scattered Tully-Fisher relation than that of field spirals.

For galaxies lying in or very near the Virgo cluster, an alternative to ram pressure gas stripping would be tidally stripped gaseous reservoirs that prevent subsequent gas infall to the disk, which quenches subsequent star formation (Larson et al. 1980). However, if a spiral galaxy lies in a cluster, it may well be on its first infall, provided that the effective ram pressure stripping of its neutral gas quickly converts it into an S0 galaxy.
It seems reasonable to expect that tidally stripped gas reservoirs will not have time to have a large effect on the gas content, so that such galaxies with tidally stripped gas reservoirs will not be as severely H I-deficient as the $133 \sigma$ cases of Table 7 .

The H I-deficient galaxies that are truly outside of the cluster may have lost their gas by the tides generated during close encounters with other galaxies within a group or small cluster of galaxies. Indeed, the efficiency of tidal perturbations of galaxies from close encounters with other galaxies is stronger in groups and small clusters than in rich clusters (see Fig. 5 of Mamon 2000), basically because the rapid motions in rich clusters decrease the efficiency of tides. Three of the four $\mathrm{H} \mathrm{I-deficient} \mathrm{galaxies} \mathrm{at} 28 \mathrm{Mpc}$ lie within a significant concentration of galaxies (Figs. 1 and 4) that ought to create such collisional tides, even with the estimates of distance using the more precise radial velocities (for these distant objects, see Eq. (12)).

The 3 galaxies outside the virial angular radius (NGC 4064, NGC 4580 and NGC 4586) all probably lie closer than 2.5 virial radii from $\mathrm{M} 87$, and may be in the process of bouncing out of the cluster after having lost their H I gas through ram pressure stripping by the intracluster gas, if the maximum rebound radius is closer to the upper limit of 2.5 virial radii found by Mamon et al. (2004), than to their lower limit of 1 virial radius. One of these galaxies (NGC 4064) is a candidate for a recent merger as witnessed by its very low inner rotation, while the other two (NGC 4580 and NGC 4586) may have interacted in the past, given their proximity in phase space. In the case of recent merging, the neutral gas may have been shock heated with that of the galaxy being swallowed up, and should shine in $\mathrm{H} \alpha$ or X-rays depending on its temperature.

Of the 4 galaxies likely or certainly far outside the Virgo cluster (in 3D), one (NGC 4305) may be tidally perturbed by a close major companion, one (NGC 4424) has suffered a recent merger (given its very low inner rotation velocity), one may have interacted with another galaxy (NGC 4307) in the past, and one (NGC 4492) may be a misclassified S0/a (with $T=0$ ), implying a lower expected HI content, hence a less than $3 \sigma$ HI-deficiency upon the estimator $\mathrm{DEF}_{1}$ and just at $3 \sigma$ upon $\mathrm{DEF}_{2}$, although the bulge/disk ratio derived by Gavazzi et al. (2000) suggests that the galaxy is indeed an Sa.

Although we can provide at least one explanation for each case of H I-deficiency in the 13 spiral galaxies that 1) appear to be located in the foreground or background of the Virgo cluster or 2) lie just outside the projected virial radius of the cluster, there is certainly room for an improved analysis. In particular, accurate distances obtained with Cepheids would be most beneficial, as well as VLA maps for 11 of the 13 galaxies to distinguish tidal stripping from ram pressure stripping.

Acknowledgements. We wish to thank Philippe Amram, Hector Bravo-Alfaro, Véronique Cayatte, Avishai Dekel, Peppo Gavazzi, Gopal Krishna, and Gilles Theureau for useful discussions, and the anonymous referee for very useful comments. We also thank François Bouchet, Bruno Guiderdoni and coworkers for kindly providing us with their $N$-body simulations, and Jeremy Blaizot for answering our technical questions about the design and access to the simulations. T.S. acknowledges hospitality of the Institut d'Astrophysique de Paris where most of this work was done, and she and G.A.M. acknowledge Ewa Łokas for hosting them at the 
CAMK in Warsaw, where part of this work was also done. T.S. was supported by a fellowship of the Ministerio de Educación, Cultura y Deporte of Spain. We have used the HyperLEDA database (http://leda.univ-lyon1.fr) operated at the Observatoire de Lyon, France, SIMBAD (http://simbad.u-strasbg . fr), operated by the CDS in Strasbourg, France, the NASA/IPAC Extragalactic Database (NED, http://nedwww.iap.caltech.edu), which is operated by the Jet Propulsion Laboratory, California Institute of Technology, under contract with the National Aeronautics and Space Administration, and the GOLDMine database operated by the Universita' degli Studi di Milano-Bicocca.

\section{References}

Bettoni, D., Galletta, G., \& García-Burillo, S. 2003, A\&A, 405, 5 Bevington, P. R., \& Robinson, D. K. 1992, Data reduction and error analysis for the physical sciences (New York: McGraw-Hill, 2nd ed.)

Binggeli, B., Sandage, A., \& Tammann, G. A. 1985, AJ, 90, 1681

Cardelli, J. A., Clayton, G. C., \& Mathis, J. S. 1989, ApJ, 345, 245

Cayatte, V., Kotanyi, C., Balkowski, C., \& van Gorkom, J. H. 1994, AJ, 107, 1003

Cayatte, V., van Gorkom, J. H., Balkowski, C., \& Kotanyi, C. 1990, AJ, 100, 604

Chamaraux, P., Balkowski, C., \& Gérard, E. 1980, A\&A, 83, 38

Cole, S., \& Lacey, C. 1996, MNRAS, 281, 716

Combes, F., Prugniel, P., Rampazzo, R., \& Sulentic, J. W. 1994, A\&A, 281, 725

Cowley, A. P., Crampton, D., \& McClure, R. D. 1982, ApJ, 263, 1

Davis, M., Efstathiou, G., Frenk, C. S., \& White, S. D. M. 1985, ApJ, 292, 371

Ekholm, T., Baryshev, Y., Teerikorpi, P., Hanski, M. O., \& Paturel, G. 2001, A\&A, 368, L17

Ekholm, T., Lanoix, P., Teerikorpi, P., Fouqué, P., \& Paturel, G. 2000, A\&A, 355, 835

Ferguson, H. C., \& Binggeli, B. 1994, A\&ARv, 6, 67

Gavazzi, G., Boselli, A., Donati, A., Franzetti, P., \& Scodeggio, M. 2003, A\&A, 400, 451

Gavazzi, G., Boselli, A., Scodeggio, M., Pierini, D., \& Belsole, E. 1999, MNRAS, 304, 595

Gavazzi, G., Franzetti, P., Scodeggio, M., Boselli, A., \& Pierini, D. 2000, A\&A, 361, 863

Giovanelli, R., \& Haynes, M. P. 1985, ApJ, 292, 404

Guiderdoni, B., \& Rocca-Volmerange, B. 1985, A\&A, 151, 108

Gunn, J. E., \& Gott, J. R. 1972, ApJ, 176, 1

Hatton, S., Devriendt, J., Ninin, S., et al. 2003, MNRAS, 343, 75
Haynes, M. P., \& Giovanelli, R. 1984, AJ, 89, 758

Haynes, M. P., \& Giovanelli, R. 1986, ApJ, 306, 466

Jensen, J. B., Tonry, J. L., \& Luppino, G. A. 1998, ApJ, 505, 111

Karachentsev, I. D., Makarov, D. I., Sharina, M. E., et al. 2003, A\&A, 398, 479

Keel, W. C. 1996, PASP, 108, 917

Kenney, J. D. P., Koopmann, R. A., Rubin, V. C., \& Young, J. S. 1996, AJ, 111, 152

Klypin, A., Hoffman, Y., Kravtsov, A. V., \& Gottlöber, S. 2003, ApJ, 596, 19

Kochanek, C. S., Pahre, M. A., Falco, E. E., et al. 2001, ApJ, 560, 566

Koopmann, R. A., \& Kenney, J. D. P. 1998, ApJ, 497, L75

Kotanyi, C. G., \& Ekers, R. D. 1983, A\&A, 122, 267

Kundu, A., \& Whitmore, B. C. 2001, AJ, 121, 2950

Larson, R. B., Tinsley, B. M., \& Caldwell, C. N. 1980, ApJ, 237, 692

Liu, C. T., \& Kennicutt, R. C. 1995, ApJ, 450, 547

Mamon, G. A. 2000, in 15th IAP Astrophys. Mtg., Dynamics of Galaxies: from the Early Universe to the Present, ed. F. Combes, G. A. Mamon, \& V. Charmandaris, Vol. 197 (San Francisco: ASP), 377 [arXiv:astro-ph/9911333]

Mamon, G. A., Sanchis, T., Salvador-Solé, E., \& Solanes, J. M. 2004, A\&A, 414, 445

Mendes de Oliveira, C., Amram, P., Plana, H., \& Balkowski, C. 2003, AJ, 126, 2635

Naim, A., Lahav, O., Buta, R. J., et al. 1995, MNRAS, 274, 1107

Neilsen, E. H., \& Tsvetanov, Z. I. 2000, ApJ, 536, 255

Ninin, S. 1999, Ph.D. Thesis, Université de Paris XI

Rubin, V. C., Hunter, D. A., Ford, W., \& Kent, J. 1991, ApJS, 76, 153

Rubin, V. C., Waterman, A. H., \& Kenney, J. D. P. 1999, AJ, 118, 236

Sanchis, T., Solanes, J. M., Salvador-Solé, E., Fouqué, P., \& Manrique, A. 2002, ApJ, 580, 164

Sandage, A., Binggeli, B., \& Tammann, G. A. 1985, AJ, 90, 1759

Schlegel, D. J., Finkbeiner, D. P., \& Davis, M. 1998, ApJ, 500, 525

Solanes, J. M., Giovanelli, R., \& Haynes, M. P. 1996, ApJ, 461, 609

Solanes, J. M., Manrique, A., García-Gómez, C., et al. 2001, ApJ, 548, 97

Solanes, J. M., Sanchis, T., Salvador-Solé, E., Giovanelli, R., \& Haynes, M. P. 2002, AJ, 124, 2440

Spergel, D. N., Verde, L., Peiris, H. V., et al. 2003, ApJS, 148, 175

Stauffer, J. R., Kenney, J. D., \& Young, J. S. 1986, AJ, 91, 1286

Teerikorpi, P., Bottinelli, L., Gouguenheim, L., \& Paturel, G. 1992, A\&A, 260, 17

Tonry, J. L., Dressler, A., Blakeslee, J. P., et al. 2001, ApJ, 546, 681

Tully, R. B., \& Fisher, J. R. 1977, A\&A, 54, 661

Tully, R. B., \& Shaya, E. J. 1984, ApJ, 281, 31

Young, C. K., \& Currie, M. J. 1995, MNRAS, 273, 1141 


\section{Online Material}




\section{Appendix A: Median and half-width of the likelihood function for a set of measurements with different errors}

For a single measurement of variable $X$ of value $x$ with uncertainty $\sigma_{i}$, assuming Gaussian errors, the probability of measuring $x_{i}$ is

$p_{i}\left(x_{i} \mid x\right)=\frac{1}{\sqrt{2 \pi} \sigma_{i}} \exp \left[-\frac{\left(x_{i}-x\right)^{2}}{2 \sigma_{i}^{2}}\right]$.

The likelihood of measuring a set of $\left\{x_{i}\right\}$ given a true value $x$ is

$$
\begin{aligned}
\mathcal{L}\left(\left\{x_{i}\right\} \mid x\right) & =\prod_{i} p_{i}\left(x_{i} \mid x\right) \\
& =\prod_{i}\left(\frac{1}{\sqrt{2 \pi} \sigma_{i}}\right) \exp \left(-\frac{S(x)}{2}\right),
\end{aligned}
$$

with

$S(x)=x^{2} \sum_{i} \frac{1}{\sigma_{i}^{2}}-2 x \sum_{i} \frac{x_{i}}{\sigma_{i}^{2}}+\sum_{i} \frac{x_{i}^{2}}{\sigma_{i}^{2}}$.

From Bayes' theorem, the probability of having a true value $x$ is

$P\left(x \mid\left\{x_{i}\right\}\right)=\frac{f(x)}{g\left(\left\{x_{i}\right\}\right)} \mathcal{L}\left(\left\{x_{i}\right\} \mid x\right)$,

where $f(x)$ is the a priori probability of having a true distance $x$ and $g\left(\left\{x_{i}\right\}\right)$ is the a priori probability of measuring the set of $\left\{x_{i}\right\}$. If the measurements are made with a selection function near unity, then $g \simeq 1$.

For simplicity, one can assume a uniform $f(x)$. The probability of the true $X$ being less than some value $x$ is then

$$
\begin{aligned}
P(<x) & =\frac{\int_{-\infty}^{x} \mathcal{L}\left(\left\{x_{i}\right\} \mid x\right) \mathrm{d} x}{\int_{-\infty}^{\infty} \mathcal{L}\left(\left\{x_{i}\right\} \mid x\right) \mathrm{d} x} \\
& =\frac{\int_{-\infty}^{x} \exp \left[-x^{2} / 2 \sum_{i} 1 / \sigma_{i}^{2}+x \sum_{i} x_{i} / \sigma_{i}^{2}\right] \mathrm{d} x}{\int_{-\infty}^{\infty} \exp \left[-x^{2} / 2 \sum_{i} 1 / \sigma_{i}^{2}+x \sum_{i} x_{i} / \sigma_{i}^{2}\right] \mathrm{d} x} \\
& =\frac{1}{2} \operatorname{erfc}\left(\frac{v}{\sqrt{2}}\right),
\end{aligned}
$$

where

$v=\frac{\sum_{i} x_{i} / \sigma_{i}^{2}-x \sum_{i} 1 / \sigma_{i}^{2}}{\sqrt{\sum_{i} 1 / \sigma_{i}^{2}}}$.

Now $\operatorname{erfc}(v / \sqrt{2}) / 2=0.16,0.5,0.84$ for $v=-1,0,+1$, so that the median $X$ is

$$
x_{50}=\frac{\sum_{i} x_{i} / \sigma_{i}^{2}}{\sum_{i} 1 / \sigma_{i}^{2}}
$$

while the width equivalent to the standard deviation of a single Gaussian is

$\sigma=\frac{1}{2}\left(x_{84}-x_{16}\right)=\frac{1}{\sqrt{\sum_{i} 1 / \sigma_{i}^{2}}}$.

Since $\mathcal{L}$ is an exponential function of $x$ (Eq. (A.2)), the mean and mode will be equal to $x_{50}$.
Alternatively, one could adopt an underlying physical model for $f(x)$. If $X$ is the distance modulus, for which the Gaussian p.d.f. of Eq. (A.1) should be adequate, one could use a Virgocentric model, where the probability $f(x)$ stems from a density distribution around Virgo, using for example an NFW model. If the angle between the galaxy and Virgo, as seen by the observer, is $\theta$, one has

$$
\begin{aligned}
r^{2}= & D_{10}^{2}\left\{\operatorname{dex}(-0.4 d)+\operatorname{dex}\left(-0.4 d_{V}\right)\right. \\
& \left.-2 \cos \theta \operatorname{dex}\left[-0.2\left(d+d_{V}\right)\right]\right\},
\end{aligned}
$$

where $d$ and $d_{V}$ are the distance moduli to the galaxy and to Virgo, respectively, and $D_{10}=10 \mathrm{pc}$. Hence, one has

$$
\begin{aligned}
f(d) \propto & \rho(r) \frac{\mathrm{d} r}{\mathrm{~d} d}, \\
\frac{\mathrm{d} r}{\mathrm{~d} d}= & \frac{1}{2 r} \frac{\mathrm{d} r^{2}}{\mathrm{~d} d} \\
= & \frac{0.2 \ln 10 D_{10}^{2}}{r} \\
& \times\left[\cos \theta \operatorname{dex}\left(-0.2\left(d+d_{V}\right)\right)-\operatorname{dex}(-0.4 d)\right] .
\end{aligned}
$$

The problem with this approach is that the underlying model, i.e. $\rho(r)$, is uncertain: should one take a single NFW model at the location of M 87? Should one make $\rho(r)$ uniform at a radius where $\rho_{\mathrm{NFW}}(r)$ reaches the mean mass density of the Universe? Or better, should one replace this uniform background with a collection of other halos, as expected in the hierarchical scenario? Moreover, because the model requires a distance to M 87, one would need to iterate using a first guess on the distance to M 87 (perhaps based upon the estimate with a uniform $f(x)$ ). These questions led us to assume in this paper a uniform distribution for $f(x)$. 